\title{
Galois extensions for coquasi-Hopf algebras
}

\author{
ADRIANA BALAN* \\ Faculty of Applied Sciences, University Politehnica of Bucharest, \\ 313 Splaiul Independenţei, 060042 Bucharest, Romania \\ e-mail: asteleanu@yahoo.com
}

April, 2008

\begin{abstract}
The notions of Galois and cleft extensions are generalized for coquasi-Hopf algebras. It is shown that such an extension over a coquasi-Hopf algebra is cleft if and only if it is Galois and has the normal basis property. A Schneider type theorem ([33]) is proven for coquasi-Hopf algebras with bijective antipode. As an application, we generalize Schauenburg's bialgebroid construction for coquasi-Hopf algebras.
\end{abstract}

\section{Introduction}

The study of Hopf Galois extensions started last century with the papers of Chase, Harrison and Rosenberg (15]) and of Chase and Sweedler ([16). Later, their definition was improved by Kreimer and Takeuchi (23]) and knew since a continuous development, mainly because their relation to different areas of mathematics. But in the last decade, examples of extensions which were not Galois but behaved like such had appeared. An explanation was necessary, and it became soon clear that this was possible only by generalization. The replacement of the Hopf algebra by a coalgebra (or more generally by a coring) has led to the notion of a Galois extension by a coalgebra, first formulated by Brzèzinski and Hajac ([9]). Another generalization was obtained considering Galois extensions over a coacting bialgebroid (over a non-commutative ground ring) ([21], 8]).

All structures cited above are generalizations of bialgebras or Hopf algebras. Another such objects are the (co)quasi-Hopf algebras. They have been introduced by Drinfeld ([19]), respectively by Majid (24]) and have lately attracted much attention in both mathematics and physics (3], 25]). So it is natural to see if it is possible to generalize the Galois theory also to the case of coquasi-Hopf algebras.

The definition of a coquasi-Hopf algebra $H$ ensures that the category of right $H$-comodules $\mathcal{M}^{H}$ is monoidal, with usual tensor product over the base field. The difference between a coquasi-Hopf algebra and a Hopf algebra is that the associativity of tensor product in the monoidal category does not coincide with the usual associativity of tensor product in the category of vector spaces. Consequently, the multiplication of a coquasi-Hopf algebra is no longer associative, but associative up to conjugation by an invertible element $\omega \in(H \otimes H \otimes H)^{*}$ (the reassociator). But is this main feature of coquasi-Hopf algebras, namely the monoidallity of corepresentations, which made possible generalizations of major properties from Hopf algebras (the existence and uniqueness of integrals, the Nichols-Zoeller Theorem, construction of the Drinfeld double, etc.). Hence it seems natural to continue with the Galois theory for coquasi-Hopf algebras.

*2000 Mathematics Subject Classification. 16W30

$\dagger$ Key words and phrases: coquasi-Hopf algebra, monoidal category, Galois extension 
The present paper begins with a short review of the known results about coquasi-Hopf algebras, their categories of comodules and about algebras and modules within these monoidal categories mentioned above. As for Hopf algebras, to each right comodule algebra $A$ (which is an algebra in the monoidal category of right comodules) one can assign a pair of adjoint functors, namely the functor of coinvariants and the induced functor. Our purpose is to generalize their well-known properties from Hopf algebras to coquasi-Hopf algebras.

In the second part it is defined the notion of Galois extension. A right comodule algebra $A$ is a Galois extension over its coinvariants ring $A^{c o H}$ (which is associative, although $A$ fails to be) if a certain map is bijective. This is a natural generalization of the author's previous paper ([6]), where only finite dimensional quasi-Hopf algebras were considered. It should be noticed that this definition for Galois extensions works only for coquasi-Hopf algebras, as it involves the presence of the antipode. Although this may look restrictive, we shall see that this definition for the Galois map allows us to recover all principal results from the classical Hopf-Galois theory. A Galois extension is invariant to any gauge transformation. As an example of Galois extension, we take a group algebra, and view it as a coquasi-Hopf algebra by a 3-cocycle. Then a comodule algebra is a graded space with a multiplication non-necessarily associative, which is Galois over its invariants if and only if it is strongly graded. This was known from long time for Hopf algebras ([35). Moreover, any crossed product $(\underline{\underline{5}})$ coming from an associative algebra endowed with a 2-cocycle and a weak action is also a Galois extension.

Recall that in the Hopf algebra case, the functor of coinvariants is a Hom functor, and the Galois map is just the evaluation for a certain relative Hopf module. We show that these results, slightly modified by the presence of a twist, hold also in the coquasi-case. We give thus an explanation for the formula of the Galois map from Definition 8. Although the results are the same, it is much more difficult to obtain them. The structure of the relative Hopf module $A \otimes H$ (which is the link between the Galois map can and the adjunction of categories $\left.\mathcal{M}_{B} \underset{(-)^{c o} H}{\stackrel{(-) \otimes_{B}}{\rightleftarrows}} \mathcal{M}_{A}^{H}\right)$ is not obvious. The classical formulas do not work anymore, and an isomorphism is required in order to get the desired structure by transport.

We introduce next the notion of a cleft extension. As this involves the convolution product (which is no longer associative), the invertibility of the cleaving map has to be translated now in relations (3.8), (3.9) involving the antipode and the linear maps $\alpha, \beta$.

As a generalization of theorems of Doi and Takeuchi ([17), and Blattner and Montgomery ([7]), we obtain the first main result of this paper, namely the equivalence between cleft extensions, and Galois extensions with the normal basis property.

The second main part of this section concerns the equivalence between the category of relative Hopf modules and modules over the subalgebra of coinvariants. It starts with an analogue of the Schneider's imprimitivity theorem of [33]. A key problem in the proof is how to show that the bijectivity of the Galois map implies the bijectivity of the corresponding map for any relative right $(A, H)$-Hopf module. As $A$ is not an associative algebra, this is not obvious and requires some special considerations about the tensor product over the algebra $A$ in the monoidal category of right comodules (Lemma 29). The proof of the theorem uses the Five Lemma applied twice to some commutative diagrams, but unlike the Hopf algebra case, the commutativity of those is not an easy fact and requires special attention care when dealing with the reassociator $\omega$ and of the elements $\alpha$ and $\beta$ (from the definition of the antipode).

Next, we prove a coquasi-version of the affineness criteria for affine algebraic groups schemes, where the surjectivity of the Galois map of the extension is related to relative injectivity of the $H$-comodule $A$ and to the equivalence between the category of relative Hopf modules and modules over the subalgebra of coinvariants.

In the last section, we generalize Schauenburg's bialgebroid construction. This is an illustration of how the Galois theory, combined with monoidally arguments can raise to new structures. 


\section{Preliminaries}

In this section we recall some definitions and results and fix notations. Throughout the paper we work over some base field $k$. Tensor products, algebras, linear spaces, etc. will be over $k$. Unadorned $\otimes$ means $\otimes_{k}$. We shall use dots to indicate the module or comodule structure on the tensor product. An introduction to the study of quasi-bialgebras and quasi-Hopf algebras and their duals (coquasi-bialgebras, respectively coquasi-Hopf algebras) can be found in 25. A good reference for monoidal categories is 222, while actions of monoidal categories are exposed in [28], [29].

Definition 1. A coquasi-bialgebra $(H, m, u, \omega, \Delta, \varepsilon)$ is a coassociative coalgebra $(H, \Delta, \varepsilon)$ together with coalgebra morphisms: the multiplication $m: H \otimes H \longrightarrow H$ (denoted $m(h \otimes g)=h g$ ), the unit $u: \mathbb{k} \longrightarrow H$ (denoted $\left.u(1)=1_{H}\right)$, and a convolution invertible element $\omega \in(H \otimes H \otimes H)^{*}$ such that:

$$
\begin{aligned}
h_{1}\left(g_{1} k_{1}\right) \omega\left(h_{2}, g_{2}, k_{2}\right) & =\omega\left(h_{1}, g_{1}, k_{1}\right)\left(h_{2} g_{2}\right) k_{2} \\
1_{H} h & =h 1_{H}=h \\
\omega\left(h_{1}, g_{1}, k_{1} l_{1}\right) \omega\left(h_{2} g_{2}, k_{2}, l_{2}\right) & =\omega\left(g_{1}, k_{1}, l_{1}\right) \omega\left(h_{1}, g_{2} k_{2}, l_{2}\right) \omega\left(h_{2}, g_{3}, k_{3}\right) \\
\omega\left(h, 1_{H}, g\right) & =\varepsilon(h) \varepsilon(g)
\end{aligned}
$$

hold for all $h, g, k, l \in H$.

As a consequence, we have also $\omega\left(1_{H}, h, g\right)=\omega\left(h, g, 1_{H}\right)=\varepsilon(h) \varepsilon(g)$ for each $g, h \in H$.

Definition 2. A coquasi-Hopf algebra is a coquasi-bialgebra $H$ endowed with a coalgebra antihomomorphism $S: H \longrightarrow H$ (the antipode) and with elements $\alpha, \beta \in H^{*}$ satisfying

$$
\begin{aligned}
S\left(h_{1}\right) \alpha\left(h_{2}\right) h_{3} & =\alpha(h) 1_{H} \\
h_{1} \beta\left(h_{2}\right) S\left(h_{3}\right) & =\beta(h) 1_{H} \\
\omega\left(h_{1} \beta\left(h_{2}\right), S\left(h_{3}\right), \alpha\left(h_{4}\right) h_{5}\right) & =\omega^{-1}\left(S\left(h_{1}\right), \alpha\left(h_{2}\right) h_{3} \beta\left(h_{4}\right), S\left(h_{5}\right)\right)=\varepsilon(h)
\end{aligned}
$$

for all $h \in H$.

These relations imply also $S\left(1_{H}\right)=1_{H}$ and $\alpha\left(1_{H}\right) \beta\left(1_{H}\right)=1$, so by rescaling $\alpha$ and $\beta$, we may assume that $\alpha\left(1_{H}\right)=1$ and $\beta\left(1_{H}\right)=1$. The antipode is unique up to a convolution invertible element $U \in H^{*}$ : if $\left(S^{\prime}, \alpha^{\prime}, \beta^{\prime}\right)$ is another triple with the above properties, then according to 25] we have

$$
S^{\prime}(h)=U\left(h_{1}\right) S\left(h_{2}\right) U^{-1}\left(h_{3}\right), \quad \alpha^{\prime}(h)=U\left(h_{1}\right) \alpha\left(h_{2}\right), \quad \beta^{\prime}(h)=\beta\left(h_{1}\right) U^{-1}\left(h_{2}\right)
$$

for all $h \in H$.

We shall use in this paper the monoidal structure of the right $H$-comodule category $\mathcal{M}^{H}$ and of the left $H$-comodule category ${ }^{H} \mathcal{M}$ : the tensor product is over the base field and the comodule structure (left or right) of the tensor product is the codiagonal one. The reassociators are

$$
\begin{aligned}
\phi_{U, V, W} & : \quad(U \otimes V) \otimes W \longrightarrow U \otimes(V \otimes W) \\
\phi_{U, V, W}((u \otimes v) \otimes w) & =u_{0} \otimes\left(v_{0} \otimes w_{0}\right) \omega\left(u_{1}, v_{1}, w_{1}\right)
\end{aligned}
$$

for $u \in U, v \in V, w \in W$ and $U, V, W \in \mathcal{M}^{H}$, respectively

$$
\begin{aligned}
\phi_{U, V, W} & : \quad(U \otimes V) \otimes W \longrightarrow U \otimes(V \otimes W) \\
\phi_{U, V, W}((u \otimes v) \otimes w) & =\omega^{-1}\left(u_{-1}, v_{-1}, w_{-1}\right) u_{0} \otimes\left(v_{0} \otimes w_{0}\right)
\end{aligned}
$$

for $u \in U, v \in V, w \in W$ and $U, V, W \in{ }^{H} \mathcal{M}$. 
Together with a coquasi-Hopf algebra with bijective antipode $H=\left(H, \Delta, \varepsilon, m, 1_{H}, \omega, S, \alpha, \beta\right)$, we also have $H^{o p}, H^{c o p}$, and $H^{o p, c o p}$ as coquasi-Hopf algebras, where "op" means opposite multiplication and "cop" means opposite comultiplication. The coquasi-Hopf structures are obtained by putting $\omega_{c o p}=\omega^{-1}, \omega_{o p}=\left(\omega^{-1}\right)^{321}$, $\omega_{\text {op }, \text { cop }}=\omega^{321}, S_{o p}=S_{\text {cop }}=\left(S_{o p, c o p}\right)^{-1}=S^{-1}, \alpha_{c o p}=\beta S^{-1}, \alpha_{o p}=\alpha S^{-1}, \alpha_{o p, c o p}=\beta, \beta_{c o p}=\alpha S^{-1}$, $\beta_{o p}=\beta S^{-1}$ and $\beta_{o p, c o p}=\alpha$. Here $\omega^{321}(h, g, k)=\omega(k, g, h)$.

For $H$ a coquasi-bialgebra, the linear dual $H^{*}=\operatorname{Hom}(H, \mathbb{k})$ becomes an associative algebra with multiplication given by the convolution product

$$
\left(h^{*} g^{*}\right)(h)=h^{*}\left(h_{1}\right) g^{*}\left(h_{2}\right) \quad \forall h \in H \quad \text { şi } \quad h^{*}, g^{*} \in H^{*}
$$

and unit $\varepsilon$. This algebra is acting on $H$ by the formulas:

$$
h^{*} \rightarrow h=h_{1} h^{*}\left(h_{2}\right), \quad h<h^{*}=h^{*}\left(h_{1}\right) h_{2}
$$

for any $h^{*} \in H^{*}, h \in H$.

Now, recall from [27] the following: for $\tau \in(H \otimes H)^{*}$ a convolution invertible map such that $\tau(1, h)=$ $\tau(h, 1)=\varepsilon(h)$ for all $h \in H$ ( $\tau$ is called a twist or a gauge transformation), one can define a new structure of coquasi-Hopf algebra on $H$, denoted $H_{\tau}$, by taking

$$
\begin{aligned}
h \cdot \tau & =\tau\left(h_{1}, g_{1}\right) h_{2} g_{2} \tau^{-1}\left(h_{3}, g_{3}\right) \\
\omega_{\tau}(h, g, k) & =\tau\left(g_{1}, k_{1}\right) \tau\left(h_{1}, g_{2} k_{2}\right) \omega\left(h_{2}, g_{3}, k_{3}\right) \tau^{-1}\left(h_{3} g_{4}, k_{4}\right) \tau^{-1}\left(h_{4}, g_{5}\right) \\
\alpha_{\tau}(h) & =\tau^{-1}\left(S\left(h_{1}\right), \alpha\left(h_{2}\right) h_{3}\right) \\
\beta_{\tau}(h) & =\tau\left(h_{1} \beta\left(h_{2}\right), S\left(h_{3}\right)\right)
\end{aligned}
$$

for all $h, g, k \in H$, and keeping the unit, the comultiplication, the counit and the antipode unchanged.

Remark 3. There is a monoidal isomorphism $\mathcal{M}^{H} \simeq \mathcal{M}^{H_{\tau}}$, which is the identity on objects and on morphisms, with monoidal structure given by $V \otimes W \longrightarrow V \otimes W, v \otimes w \longrightarrow v_{0} \otimes w_{0} \tau^{-1}\left(v_{1}, w_{1}\right)$, where $v \in V$, $w \in W$ and $V, W \in \mathcal{M}^{H}$.

In [11, it was constructed a twist $f \in(H \otimes H)^{*}$ which controls how far is $S$ from a anti-algebra morphism:

$$
\mathbf{f}\left(h_{1}, g_{1}\right) S\left(h_{2} g_{2}\right)=S\left(g_{1}\right) S\left(h_{1}\right) \mathbf{f}\left(h_{2}, g_{2}\right)
$$

If we denote

$$
\begin{aligned}
& p(h, g)=\omega\left(S\left(g_{2}\right), S\left(h_{2}\right), h_{4}\right) \omega^{-1}\left(S\left(g_{1}\right) S\left(h_{1}\right), h_{5}, g_{4}\right) \alpha\left(h_{3}\right) \alpha\left(g_{3}\right) \\
& q(h, g)=\omega\left(h_{1} g_{1}, S\left(g_{5}\right), S\left(h_{4}\right)\right) \omega^{-1}\left(h_{2}, g_{2}, S\left(g_{4}\right)\right) \beta\left(h_{3}\right) \beta\left(g_{3}\right)
\end{aligned}
$$

then the twist $\mathbf{f}$ is given by

$$
\mathbf{f}(h, g)=\omega^{-1}\left(S\left(g_{1}\right) S\left(h_{1}\right), h_{3} g_{3}, S\left(h_{5} g_{5}\right)\right) p\left(h_{2}, g_{2}\right) \beta\left(h_{4} g_{4}\right)
$$

We have also that

$$
\begin{aligned}
\mathbf{f}\left(h_{1}, g_{1}\right) \alpha\left(h_{2} g_{2}\right) & =p(h, g) \\
\beta\left(h_{1} g_{1}\right) \mathbf{f}^{(-1)}\left(h_{2}, g_{2}\right) & =q(h, g) \\
p\left(h_{1}, S\left(h_{3}\right)\right) \beta\left(h_{2}\right) & =\alpha S(h) \\
\mathbf{f}\left(h_{1}, S\left(h_{3}\right)\right) \beta\left(h_{2}\right)= & \alpha S(h) \\
\mathbf{f}^{(-1)}\left(S^{-1}\left(g_{1}\right), S^{-1}\left(h_{1}\right)\right) \omega^{-1}\left(g_{4}, \alpha S^{-1}\left(g_{3}\right) S^{-1}\left(g_{2}\right), S^{-1}\left(h_{2}\right)\right)= & \mathbf{f}\left(g_{5}, S^{-1}\left(h_{1} g_{1}\right)\right) \\
& \omega^{\cdot 1}\left(h_{2}, g_{2} \beta\left(g_{3}\right), S\left(g_{4}\right)\right)
\end{aligned}
$$


where in the last formula we assumed the bijectivity of the antipode. Relations (2.18) and (2.19) are from [14, (2.20) is an easy consequence of the formula of $p$, (2.21) follows immediately from (2.20), while for (2.22) we use (2.12) and the fact that the associator $\omega_{\mathbf{f}}$ for the twisted coquasi-bialgebra $H_{\mathbf{f}}$ is $\omega_{\mathbf{f}}(h, g, k)=$ $\omega(S(k), S(g), S(h)), \forall h, g, k \in H$. If the antipode is bijective, then by passing from $H$ to $H^{o p}$ we obtain a new twist $\widetilde{\mathbf{f}} \in(H \otimes H)^{*}[12$, given by

$$
\widetilde{\mathbf{f}}(h, g)=\mathbf{f}\left(S^{-1}(g), S^{-1}(h)\right)
$$

which satisfies

$$
\widetilde{\mathbf{f}}\left(h_{1}, g_{1}\right) S^{-1}\left(h_{2} g_{2}\right)=S^{-1}\left(g_{1}\right) S^{-1}\left(h_{1}\right) \widetilde{\mathbf{f}}\left(h_{2}, g_{2}\right)
$$

for any $h, g \in H$. The corresponding reassociator will be $\omega_{\widetilde{\mathbf{f}}}(h, g, k)=\omega\left(S^{-1}(k), S^{-1}(g), S^{-1}(h)\right)$. This twist will appear later.

Definition 4. ([13]) A right $H$-comodule algebra $A$ is an algebra in the monoidal category $\mathcal{M}^{H}$. This means $\left(A, \rho_{A}\right)$ is a right $H$-comodule, we have a multiplication map $m_{A}: A \otimes A \longrightarrow A$, denoted $m_{A}(a \otimes b)=a b$, for $a, b \in A$, and a unit map $u_{A}: \mathbb{k} \longrightarrow A$, where we put $u_{A}(1)=1_{A}$, which are both $H$-colinear, such that

$$
(a b) c=a_{0}\left(b_{0} c_{0}\right) \omega\left(a_{1}, b_{1}, c_{1}\right)
$$

holds for any $a, b, c \in A$.

Similarly we may define a left $H$-comodule algebra as an algebra in ${ }^{H} \mathcal{M}$. Notice that $A$ is a right $H$-comodule algebra if and only if $A^{o p}$ is a left $H^{o p, c o p}$-comodule algebra.

Definition 5. ([13]) For $A$ a right $H$-comodule algebra, we may define the notion of right module over $A$ in the category $\mathcal{M}^{H}$. Explicitly, this is a right $H$-comodule $\left(M, \rho_{M}\right)$, endowed with a right $A$-action, denoted $\mu_{M}(m, a)=m a$, such that

$$
\begin{aligned}
(m a) b & =m_{0}\left(a_{0} b_{0}\right) \omega\left(m_{1}, a_{1}, b_{1}\right) \\
m 1_{A} & =m \\
\rho_{M}(m a) & =m_{0} a_{0} \otimes m_{1} a_{1}
\end{aligned}
$$

hold for all $m \in M, a, b \in A$. The category of such objects, with morphisms the right $H$-colinear maps which respect the A-action, is called the category of relative right $(H, A)$-Hopf modules and denoted $\mathcal{M}_{A}^{H}$.

In the same way, we may define the category of left relative Hopf modules ${ }_{A} \mathcal{M}^{H}$ for $A$ a right $H$-comodule algebra. If $A$ is a left $H$-comodule algebra we can define similarly the categories ${ }_{A}^{H} \mathcal{M}$ and ${ }^{H} \mathcal{M}_{A}$. For later use, remark that the following categories are isomorphic:

$$
{ }_{A}^{H} \mathcal{M} \simeq \mathcal{M}_{A^{o p}}^{H^{o p, c o p}}
$$

for any $A$ a left $H$-comodule algebra ([13]).

Remark 6. It was proven in [13] that if $\tau$ is a twist on $H$, then the formula

$$
a \cdot_{\tau} b=a_{0} b_{0} \tau^{-1}\left(a_{1}, b_{1}\right)
$$

for all $a, b \in A$ defines a new multiplication such that $A$, with this new multiplication (denoted $A_{\tau-1}$ ) becomes a right $H_{\tau}$-comodule algebra. It is easy to see that the isomorphism of Remark 3 sends the algebra $A$ of the monoidal category $\mathcal{M}^{H}$ exactly to the algebra $A_{\tau^{-1}}$ in $\mathcal{M}^{H_{\tau}}$. But $\mathcal{M}^{H}$ and $\mathcal{M}^{H_{\tau}}$ are monoidally isomorphic, therefore the categories of right relative Hopf modules $\mathcal{M}_{A}^{H}$ and $\mathcal{M}_{A_{\tau-1}}^{H_{\tau}}$ will also be isomorphic. 
Let $A$ be a right $H$-comodule algebra. Consider the space of coinvariants

$$
B=A^{c o H}=\left\{a \in A \mid \rho_{A}(a)=a \otimes 1_{H}\right\}
$$

It is immediate that this is an associative algebra with unit and multiplication induced by the unit and the multiplication of $A$.

Now for each $M \in \mathcal{M}_{A}^{H}$, denote $M^{c o H}=\left\{m \in M \mid \rho_{M}(m)=m \otimes 1_{H}\right\}$. Then $M^{c o H}$ becomes naturally a right $B$-module, so we get the coinvariant functor

$$
\mathcal{M}_{A}^{H} \stackrel{(-)^{c o H}}{\longrightarrow} \mathcal{M}_{B}
$$

Notice also the natural isomorphism

$$
\operatorname{Hom}_{A}^{H}(A, M) \simeq M^{c o H}
$$

for any $M \in \mathcal{M}_{A}^{H}$. Conversely, for $N \in \mathcal{M}_{B}$, we have $N \otimes_{B} A \in \mathcal{M}_{A}^{H}$ by

$$
\begin{aligned}
\rho\left(n \otimes_{B} a\right) & =n \otimes_{B} a_{0} \otimes a_{1} \\
\left(n \otimes_{B} a\right) b & =n \otimes_{B} a b
\end{aligned}
$$

As in the classical Hopf algebra case, we obtain the following:

Proposition 7. The induced functor $(-) \otimes_{B} A$ is a left adjoint for the functor of coinvariants $(-)^{c o H}$ :

$$
\mathcal{M}_{B} \underset{(-)^{c o H}}{\stackrel{(-) \otimes_{B}}{\rightleftarrows}} \mathcal{M}_{A}^{H}
$$

Proof. Straightforward. For later use, we mention the adjunction morphisms:

$$
\begin{aligned}
& \varepsilon_{M}: M^{c o H} \otimes_{B} A \longrightarrow M, \quad \varepsilon_{M}\left(m \otimes_{B} a\right)=m a \\
& u_{N}: N \longrightarrow\left(N \otimes_{B} A\right)^{c o H}, \quad u_{N}(n)=n \otimes_{B} 1_{A}
\end{aligned}
$$

for each $N \in \mathcal{M}_{B}$ and $M \in \mathcal{M}_{A}^{H}$. Using the isomorphism form relation 2.28, we get that the counit of the adjunction is simply the evaluation.

Similarly we could define the left version of the adjunction between the induced and the coinvariant functor, namely $\mathcal{M}_{B} \underset{(-)^{\text {co } H}}{\stackrel{A \otimes_{B}(-)}{\rightleftarrows}} A \mathcal{M}^{H}$.

In the next section we shall see necessary and sufficient conditions for these adjunctions to be equivalences.

\section{Galois extensions}

Let $H$ be a coquasi-Hopf algebra with antipode $S$ and $A$ a right $H$-comodule algebra. Denote as before $B=A^{c o H}$.

Definition 8. The extension $B \subseteq A$ is $(H, S)$-Galois if the map can c $_{S} A \otimes_{B} A \longrightarrow A \otimes H$, given by

$$
a \otimes_{B} b \longrightarrow a_{0} b_{0} \otimes b_{4} \omega^{-1}\left(a_{1}, b_{1} \beta\left(b_{2}\right), S\left(b_{3}\right)\right)
$$

is bijective. 
Remark 9. (1) Although $A$ is not an associative algebra, we still keep the expression "extension $B \subseteq A$ ".

(2) Recall that for coquasi-Hopf algebras the antipode is unique up to conjugation to an invertible element. Therefore we need to check what is happening if we change $S$. Consider another triple $\left(S^{\prime}, \alpha^{\prime}, \beta^{\prime}\right)$ given by a convolution invertible element $U \in H^{*}$, as in (2.8). Then we have

$$
\begin{aligned}
\operatorname{can}_{S^{\prime}}\left(a \otimes_{B} b\right) & =a_{0} b_{0} \otimes \omega^{-1}\left(a_{1}, b_{1} \beta^{\prime}\left(b_{2}\right), S^{\prime}\left(b_{3}\right)\right) b_{4} \\
& =a_{0} b_{0} \otimes \omega^{-1}\left(a_{1}, b_{1} \beta\left(b_{2}\right) U^{-1}\left(b_{3}\right), U\left(b_{4}\right) S\left(b_{5}\right) U^{-1}\left(b_{6}\right)\right) b_{7} \\
& =a_{0} b_{0} \otimes \omega^{-1}\left(a_{1}, b_{1} \beta\left(b_{2}\right), S\left(b_{3}\right)\right) U^{-1}\left(b_{4}\right) b_{5}
\end{aligned}
$$

for every $a, b \in A$. If we define the linear map $\psi_{U}: A \otimes H \longrightarrow A \otimes H, a \otimes h \longrightarrow a \otimes U\left(h_{1}\right) h_{2}$, it is easy to see that this is bijective with inverse $a \otimes h \longrightarrow a \otimes U^{-1}\left(h_{1}\right) h_{2}$ and that $\operatorname{can}_{S^{\prime}}=\psi_{U} \circ c a n_{S}$; therefore the two Galois maps will be simultaneously bijective. In the sequel, we shall fix the antipode $S$ and the elements $\alpha$, $\beta$, such that $\alpha(1)=\beta(1)=1$, and write simply can.

In case of a Hopf algebra, the coassociator $\omega$ and the linear map $\beta$ vanish, and we recover the usual definition of the Galois map. But unlike the Hopf case, notice this time the presence of the antipode in the formula of can, which implies that this definition is possible only for coquasi-Hopf algebras, not also for coquasi-bialgebras. However, we shall see that this definition for the Galois map allows us to recover all principal results from the classical Hopf-Galois theory. In [26, Masuoka uses the classical definition of the can map, $a \otimes_{B} b \longrightarrow a b_{0} \otimes b_{1}$, to show that a certain extension is Galois over a given coquasi-Hopf algebra (which is a bicrossed product associated to some cocyle data). It is only a matter of computation to see that in the quoted case, the formula (3.1) reduces to $a \otimes_{B} b \longrightarrow a b_{0} \otimes b_{1}$. Therefore [26] provides us a first example of a non-trivial Galois extension over a coquasi-Hopf algebra.

Example 10. ([2]) Let $G$ be any group and $\omega: G \times G \times G \longrightarrow \mathbb{k}$ an invertible normalized cocycle. The the usual group algebra $H=\mathbb{k} G$ becomes a coquasi-Hopf algebra by keeping the ordinary operations, but with coassociator $\omega$ (linearly extended to $\mathbb{k} G^{\otimes 3}$ ) and linear maps $\alpha=\varepsilon$ and $\beta$ given by $\beta(g)=\omega^{-1}\left(g, g^{-1}, g\right)$, for any $g \in G$. As the coalgebra structure is not modified, a $\mathbb{k} G$-coaction means precisely a $G$-graduation. Therefore, the notion of an $H$-comodule algebra becomes in this case: a $G$-graded vector space $A=\oplus_{g \in G} A_{g}$, endowed with a unit and a multiplication " ." : $A \otimes A \rightarrow A$ such that $A_{g} A_{h} \subseteq A_{g h}$ for all $g, h \in G$, and associative in the sense that

$$
(a \cdot b) \cdot c=a \cdot(b \cdot c) \omega(|a|,|b|,|c|)
$$

for all homogeneous elements $a, b, c \in A$. The coinvariants $A^{c o H}$ are exactly $A_{e}$, where $e$ is the neutral element of $G$. We have then the following:

Proposition 11. The extension $A_{e} \subseteq A$ is Galois (in the sense of Definition 8) if and only if it is strongly graded.

Proof. Notice first that $A$ is strongly graded $\Longleftrightarrow A_{g} A_{g^{-1}}=A_{e}$ for any $g \in G$. One inclusion is obvious, and for the other we shall use the associativity rule of $A$ :

$$
\begin{aligned}
A_{g h} & \subseteq A_{g h} A_{e} \subseteq A_{g h}\left(A_{h^{-1}} A_{h}\right)=\left(A_{g h} A_{h^{-1}}\right) A_{h} \omega^{-1}\left(g h, h^{-1}, h\right) \\
& \subseteq A_{g} A_{h} \omega^{-1}\left(g h, h^{-1}, h\right) \subseteq A_{g} A_{h}
\end{aligned}
$$

Now the proof follows as in the Hopf case.

This result generalizes the Ulbrich's well-known example in the Hopf algebra case ([35]), and it is the first confirmation of the fact that our definition of a Galois extension is the correct one. 
Example 12. Another example of Galois extension can be found in [5]. Starting from a coquasi-Hopf algebra $H$ and an associative algebra $R$ endowed with an $H$-weak action and a 2-cocycle $\sigma: H \otimes H \longrightarrow R$, we can construct the crossed product $R \#_{\sigma} H$, generalizing the Hopf case. This is a Galois extension of $R$ in the sense of Definition 8, Also, the Galois extension mentioned above from [26] is precisely a particular case of our crossed product construction.

Now, remember that a Hopf algebra $H$ can be seen as a right $H$-comodule algebra via $\Delta$ and usual multiplication. The coinvariants are $\mathbb{k} 1_{H} \simeq \mathbb{k}$. Moreover, this extension is $H$-Galois (by [20]) (actually, any bialgebra $H$ is a comodule algebra in this way, and it is a Hopf algebra if and only if it is Galois). Now, working with a coquasi-Hopf algebra $H$ still gives us a right comodule, but no longer an algebra in the monoidal category $\mathcal{M}^{H}$ with the usual multiplication. If we try to deform the multiplication on $H$ via a twist $\tau$ as in (2.27), then $\left(H, \bullet_{\tau}, \Delta\right)$ is a right $H$-comodule algebra if and only if $\omega_{\tau}$ is trivial, i.e. $H_{\tau}$ is a Hopf algebra. It is unclear to the author for the moment which multiplication structure should be defined on $H$ such that we get a right $H$-comodule algebra, which in the Hopf case should reduce to ordinary multiplication. Moreover, this new multiplication should provide an example of Galois extension $\mathbb{k} \subseteq H$.

Remark 13. Let $H$ a coquasi-Hopf algebra with bijective antipode and $A$ a right $H$-comodule algebra. Notice that $\mathcal{M}^{H} \simeq{ }^{H^{c o p}} \mathcal{M}$ as monoidal categories. Using also the isomorphism from (2.26), it follows that ${ }_{A} \mathcal{M}^{H} \simeq \mathcal{M}_{A^{o p}}^{H^{o p}}$ for $A$ a right $H$-comodule algebra. For completeness, we remark that the corresponding Galois map for $H^{o p}$-extension $B^{o p} \subseteq A^{o p}$ will be

$$
\operatorname{can}^{\prime}\left(a \otimes_{B} b\right)=a_{0} b_{0} \otimes a_{4} \omega\left(S^{-1}\left(a_{3}\right) \beta S^{-1}\left(a_{2}\right), a_{1}, b_{1}\right)
$$

Then we get:

Lemma 14. The map can' is bijective if and only if can is bijective.

Proof. Consider the map $\Xi: A \otimes H \longrightarrow A \otimes H, \Xi(a \otimes h)=a_{0} \otimes a_{3} S\left(h_{1}\right) \omega^{-1}\left(h_{3}, a_{2} \beta\left(a_{3}\right), S\left(a_{4}\right)\right) \mathbf{f}\left(h_{2}, S^{-1}\left(a_{1}\right)\right)$. Then $\Xi \circ c a n=c a n^{\prime}$. Also one may check that $\Xi$ is bijective, with inverse given by

$$
\Xi^{-1}(a \otimes h)=a_{0} \otimes S^{-1}\left(h_{1}\right) a_{5} \mathbf{f}^{-1}\left(S^{-1}\left(h_{2}\right), a_{4}\right) \omega\left(a_{1} \beta\left(a_{2}\right), S\left(a_{3}\right), h_{3}\right)
$$

Notice that in the case of a Hopf algebra the map $c a n^{\prime}$ reduces to the usual formula $a \otimes_{B} b=a_{0} b \otimes_{B} a_{1}$.

Remark 15. If $A$ is a right $H$-comodule algebra and $\tau$ a twist for $H$, we may consider the twisted comodule algebra $A_{\tau^{-1}}$ as in Remark 6. The comodule structure being the same, $A$ and $A_{\tau^{-1}}$ will have same coinvariants $B$ (but over different coquasi-Hopf algebras). Then we have the following:

Proposition 16. The extension $B \subseteq A$ is $H$-Galois if and only if $B \subseteq A_{\tau^{-1}}$ is $H_{\tau^{-}}$-Galois.

Proof. The canonical Galois map for the extension $B \subseteq A_{\tau^{-1}}$ is

$$
\begin{aligned}
\operatorname{can}_{\tau}\left(a \otimes_{B} b\right)= & a_{0} \cdot_{\tau} b_{0} \otimes \omega_{\tau}^{-1}\left(a_{1}, b_{1} \beta_{\tau}\left(b_{2}\right), S\left(b_{3}\right)\right) b_{4} \\
(\underline{2.27}),(2.12),(2.14)= & a_{0} b_{0} \tau^{-1}\left(a_{1}, b_{1}\right) \otimes \tau\left(a_{2}, b_{2}\right) \tau\left(a_{3} b_{3}, S\left(b_{13}\right)\right) \omega^{-1}\left(a_{4}, b_{4}, S\left(b_{12}\right)\right) \\
& \tau^{-1}\left(a_{5}, b_{5} S\left(b_{11}\right)\right) \tau^{-1}\left(b_{6}, S\left(b_{10}\right)\right) b_{14} \tau\left(b_{7} \beta\left(b_{8}\right), S\left(b_{9}\right)\right) \\
= & a_{0} b_{0} \otimes \tau\left(a_{1} b_{1}, S\left(b_{5}\right)\right) \omega^{-1}\left(a_{2}, b_{2}, \beta\left(b_{3}\right) S\left(b_{4}\right)\right) b_{6}
\end{aligned}
$$

for any $a, b \in A$. Consider now the linear map

$$
\vartheta: A \otimes H \longrightarrow A \otimes H, \vartheta(a \otimes h)=a_{0} \otimes h_{2} \tau\left(a_{1}, S\left(h_{1}\right)\right)
$$

It is easy to check that $\vartheta$ is bijective, with inverse $\vartheta^{-1}(a \otimes h)=a_{0} \otimes h_{2} \tau^{-1}\left(a_{1}, S\left(h_{1}\right)\right)$. Then the following relation hold: $\operatorname{can}_{\tau}=\vartheta \circ \mathrm{can}$, which tells us that both extensions will be simultaneously Galois. 
It follows from the previous Remark that if $H$ is a Hopf algebra and $B \subseteq A$ is a $H$-Galois extension in the classical sense, then for any nontrivial twist $\tau \in(H \otimes H)^{*}$, the extension $B \subseteq A_{\tau^{-1}}$ will be $H_{\tau^{-}}$Galois in the sense of our Definition. Hence all known examples of Hopf-Galois extensions fit in our picture.

Example 17. Again, let $G$ a group and $\tau: G \times G \longrightarrow \mathbb{k}$ an invertible normalized map. Then $H=\mathbb{k} G$ is a Hopf algebra and $A=\mathbb{k} G$ is an $H$-comodule algebra via comultiplication. Using the twist obtained by

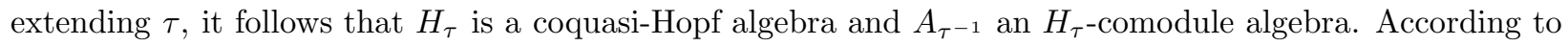
Remark 15, $A_{\tau^{-1}}$ will be a Galois extension of $\mathbb{k}$. In particular, taking $G=\left(\mathbb{Z}_{2}\right)^{n}$, it follows that all Cayley algebras (as in [1]) are Galois extensions over a coquasi-Hopf algebra.

Remark 18. In the Hopf algebra case, the Galois map arises naturally as the evaluation map $\operatorname{Hom}_{A}^{H}(A, A \otimes$ $H) \otimes_{B} A \longrightarrow A \otimes H$, from the adjunction between the induced and the coinvariant functor, applied to the relative Hopf module $A_{\bullet} \otimes H_{\bullet}$. We shall see that a similar result holds here, explaining thus the formula chosen for can. We need first some work. For the beginning, it is not obvious which $(H, A)$-Hopf module structure can be defined on $A \otimes H$ to generalize the one in the Hopf case. We shall assume that the antipode of $H$ is bijective, and obtaining the following:

Lemma 19. The map

$$
\begin{aligned}
\eta & : \quad H^{\bullet} \otimes A^{\bullet} \longrightarrow A \otimes H^{\bullet}, \\
h \otimes a & \longrightarrow \quad a_{0} \otimes \omega\left(h_{1}, a_{3}, \alpha S^{-1}\left(a_{2}\right) S^{-1}\left(a_{1}\right)\right) h_{2} a_{4}
\end{aligned}
$$

is a right $H$-comodule isomorphism, where $H \otimes A$ is a comodule via the codiagonal structure (i.e. $\rho_{H \otimes A}(h \otimes$ $\left.a)=h_{1} \otimes a_{0} \otimes h_{2} a_{1}\right)$ and $A \otimes H$ has the induced comodule structure from the one of $H$.

Proof. The inverse for $\eta$ is given by $\eta^{-1}(a \otimes h)=h_{1} S^{-1}\left(a_{4}\right) \otimes a_{0} \omega^{-1}\left(h_{2}, S^{-1}\left(a_{3}\right) \beta S^{-1}\left(a_{2}\right), a_{1}\right)$.

Corollary 20. Via the previous isomorphism, $A \otimes H$ becomes a right $A$-module in $\mathcal{M}^{H}$.

Proof. As $H$ is a right $H$-comodule via $\Delta, H^{\bullet} \otimes A_{\bullet}^{\bullet}$ is naturally the right $A$-module induced in $\mathcal{M}^{H}$, with structures

$$
\begin{aligned}
\rho_{H \otimes A}(h \otimes a) & =h_{1} \otimes a_{0} \otimes h_{2} a_{1} \\
(h \otimes a) b & =h_{1} \otimes a_{0} b_{0} \omega\left(h_{2}, a_{1}, b_{1}\right)
\end{aligned}
$$

for all $h \in H, a, b \in A$. Using $\eta$, the $A$-module structure can be transferred on $A \otimes H$. Let's see how the multiplication formula with elements of $A$ looks like:

$$
\begin{aligned}
(a \otimes h) \otimes b \longrightarrow & {\left[h_{1} S^{-1}\left(a_{4}\right) \otimes a_{0} \omega^{-1}\left(h_{2}, S^{-1}\left(a_{3}\right) \beta S^{-1}\left(a_{2}\right), a_{1}\right)\right] b } \\
= & h_{1} S^{-1}\left(a_{6}\right) \otimes a_{0} b_{0} \omega\left(h_{2} S^{-1}\left(a_{5}\right), a_{1}, b_{1}\right) \omega^{-1}\left(h_{3}, S^{-1}\left(a_{4}\right) \beta S^{-1}\left(a_{3}\right), a_{2}\right) \\
= & h_{1} S^{-1}\left(a_{6}\right) \otimes a_{0} b_{0} \omega^{-1}\left(h_{2}, S^{-1}\left(a_{5}\right), a_{1} b_{1}\right) \omega\left(S^{-1}\left(a_{4}\right), a_{2}, b_{2}\right) \beta S^{-1}\left(a_{3}\right) \\
\longrightarrow & a_{0} b_{0} \otimes\left[h_{2} S^{-1}\left(a_{10}\right)\right]\left(a_{4} b_{4}\right) \\
& \omega\left(h_{1} S^{-1}\left(a_{11}\right), a_{3} b_{3}, \alpha S^{-1}\left(a_{2} b_{2}\right) S^{-1}\left(a_{1} b_{1}\right)\right) \\
& \omega^{-1}\left(h_{3}, S^{-1}\left(a_{9}\right), a_{5} b_{5}\right) \omega\left(S^{-1}\left(a_{8}\right), a_{6}, b_{6}\right) \beta S^{-1}\left(a_{7}\right) \\
= & a_{0} b_{0} \otimes h_{3} b_{6} \omega\left(S^{-1}\left(a_{7}\right), a_{5}, b_{5}\right) \omega^{-1}\left(h_{2}, S^{-1}\left(a_{8}\right), a_{4} b_{4}\right) \\
& \omega\left(h_{1} S^{-1}\left(a_{9}\right), a_{3} b_{3}, S^{-1}\left(a_{1} b_{1}\right)\right) \alpha S^{-1}\left(a_{2} b_{2}\right) \beta S^{-1}\left(a_{6}\right) \\
\text { (2.1), (2.1), (2.6) } & a_{0} b_{0} \otimes h_{2} b_{7} \omega\left(S^{-1}\left(a_{10}\right), a_{4} b_{4}, S^{-1}\left(a_{2} b_{2}\right)\right) \omega\left(h_{1}, S^{-1}\left(a_{9}\right)\left(a_{5} b_{5}\right),\right. \\
& \left.S^{-1}\left(a_{1} b_{1}\right)\right) \omega\left(S^{-1}\left(a_{8}\right), a_{6}, b_{6}\right) \alpha S^{-1}\left(a_{3} b_{3}\right) \beta S^{-1}\left(a_{7}\right) \\
\text { (2.1) }, \text { (2.5) } & a_{0} b_{0} \otimes h_{2} b_{7} \omega\left(S^{-1}\left(a_{8}\right), a_{4} b_{4}, S^{-1}\left(a_{2} b_{2}\right)\right) \omega\left(S^{-1}\left(a_{7}\right), a_{5}, b_{5}\right) \\
& \text { (2.3) }
\end{aligned}
$$




$$
\omega\left(h_{1}, b_{6}, S^{-1}\left(a_{1} b_{1}\right)\right) \alpha S^{-1}\left(a_{3} b_{3}\right) \beta S^{-1}\left(a_{6}\right)
$$

Therefore, we have

$$
\begin{aligned}
(a \otimes h) b= & a_{0} b_{0} \otimes h_{2} b_{7} \omega\left(S^{-1}\left(a_{8}\right), a_{4} b_{4}, \alpha S^{-1}\left(a_{3} b_{3}\right) S^{-1}\left(a_{2} b_{2}\right)\right) \omega\left(\beta S^{-1}\left(a_{6}\right) S^{-1}\left(a_{7}\right), a_{5}, b_{5}\right) \\
& \omega\left(h_{1}, b_{6}, S^{-1}\left(a_{1} b_{1}\right)\right)
\end{aligned}
$$

for any $h \in H, a, b \in A$. Seems to be complicated, but in the Hopf algebra case it simply reduces to $(a \otimes h) b=a b_{0} \otimes h b_{1}$.

We come back now to the counit of the adjunction, applied to the Hopf module $A \otimes H$. The coinvariants are $(A \otimes H)^{c o H}=A \otimes \mathbb{k} 1_{H} \simeq A$, as the coaction takes place only on the second component and $\mathbb{k}$ is a commutative field. Hence

$$
\begin{aligned}
\varepsilon_{A \otimes H}: & A \otimes_{B} A \longrightarrow A \otimes H \\
\varepsilon_{A \otimes H}\left(a \otimes_{B} b\right)= & a_{0} b_{0} \otimes b_{5} \omega\left(S^{-1}\left(a_{7}\right), a_{3} b_{3}, \alpha S^{-1}\left(a_{2} b_{2}\right) S^{-1}\left(a_{1} b_{1}\right)\right) \\
& \omega\left(\beta S^{-1}\left(a_{5}\right) S^{-1}\left(a_{6}\right), a_{4}, b_{4}\right) \\
(2.24)= & a_{0} b_{0} \otimes b_{7} \omega\left(S^{-1}\left(a_{9}\right), a_{5} b_{5}, S^{-1}\left(b_{2}\right) S^{-1}\left(a_{2}\right)\right) \omega\left(S^{-1}\left(a_{8}\right), a_{6}, b_{6}\right) \\
& \alpha\left(S^{-1}\left(b_{3}\right) S^{-1}\left(a_{3}\right)\right) \beta S^{-1}\left(a_{7}\right) \mathbf{f}^{(-1)}\left(S^{-1}\left(b_{1}\right), S^{-1}\left(a_{1}\right)\right) \\
& \mathbf{f}\left(S^{-1}\left(b_{4}\right), S^{-1}\left(a_{4}\right)\right) \\
= & a_{0} b_{0} \otimes b_{10} \mathbf{f}^{(-1)}\left(S^{-1}\left(b_{1}\right), S^{-1}\left(a_{1}\right)\right) \beta S^{-1}\left(a_{9}\right) \alpha S^{-1}\left(b_{5}\right) \alpha S^{-1}\left(a_{4}\right) \\
& \omega\left(a_{5}, b_{6}, S^{-1}\left(b_{4}\right)\right) \omega^{-1}\left(a_{6} b_{7}, S^{-1}\left(b_{3}\right), S^{-1}\left(a_{3}\right)\right) \\
& \omega\left(S^{-1}\left(a_{11}\right), a_{7} b_{8}, S^{-1}\left(b_{2}\right) S^{-1}\left(a_{2}\right)\right) \omega\left(S^{-1}\left(a_{10}\right), a_{8}, b_{9}\right) \\
= & a_{0} b_{0} \otimes b_{10} \mathbf{f}^{(-1)}\left(S^{-1}\left(b_{1}\right), S^{-1}\left(a_{1}\right)\right) \beta S^{-1}\left(a_{9}\right) \alpha S^{-1}\left(b_{5}\right) \alpha S^{-1}\left(a_{4}\right) \\
& \omega\left(S^{-1}\left(a_{12}\right), a_{5}, S^{-1}\left(a_{3}\right)\right) \omega\left(a_{6}, b_{6}, S^{-1}\left(b_{4}\right)\right) \\
& \omega\left(S^{-1}\left(a_{11}\right), a_{7} b_{7}, S^{-1}\left(b_{3}\right)\right) \omega\left(S^{-1}\left(a_{10}\right), a_{8}, b_{8}\right) \\
& \omega^{-1}\left(b_{9}, S^{-1}\left(b_{2}\right), S^{-1}\left(a_{2}\right)\right) \\
= & a_{0} b_{0} \otimes b_{5} \mathbf{f}^{(-1)}\left(S^{-1}\left(b_{1}\right), S^{-1}\left(a_{1}\right)\right) \alpha S^{-1}\left(b_{3}\right) \\
& \omega\left(b_{4}, S^{-1}\left(b_{2}\right), S^{-1}\left(a_{2}\right)\right) \\
= & a_{0} b_{0} \otimes b_{6} \mathbf{f}\left(b_{5}, S^{-1}\left(a_{1} b_{1}\right)\right) \omega^{-1}\left(a_{2}, b_{2} \beta\left(b_{3}\right), S\left(b_{4}\right)\right) \\
\text { (2.1), (2.5), (2.5), (2.6), (2.7) (2.2. (2.2) } &
\end{aligned}
$$

But according to (3.3), this is precisely can $_{\tilde{\mathbf{f}}}$, the Galois map twisted by $\widetilde{\mathbf{f}}$, where the twist $\widetilde{\mathbf{f}}$ was introduced in relation (2.23). From the Remark[15] it follows that:

Corollary 21. The bijectivity of $\varepsilon_{A \otimes H}$ implies that $B \subseteq A$ is Galois.

Remark 22. We could had used formula 3.6 as an alternative Galois map, but we preferred the formula from Definition 8 to avoid the presence of the twist and simplify computations.

We shall further need some properties of the Galois map, analogs to those in [34]:

Proposition 23. The morphism can satisfies the following:

(1) It is right $H$-colinear, where the right comodule structure on both spaces is given from the second tensorand.

(2) For any $a \in A, \operatorname{can}\left(1 \otimes_{B} a\right)=a_{0} \otimes \beta\left(a_{1}\right) a_{2}$.

(3) It is also right $H$-colinear, but with respect to the following coactions: $\hat{\rho}\left(a \otimes_{B} b\right)=a_{0} \otimes_{B} b \otimes a_{1}$, for $a \otimes_{B} b \in A \otimes_{B} A$, respectively $\check{\rho}(a \otimes h)=a_{0} \otimes h_{2} \otimes a_{1} S\left(h_{1}\right)$, where $a \otimes h \in A \otimes H$. 
(4) If can is bijective, then

$\left(c \otimes 1_{H}\right) \operatorname{can}^{-1}(d \otimes h)=\operatorname{can}^{-1}\left(c_{0} d_{0} \otimes h_{2}\right) \omega^{-1}\left(c_{1}, d_{1}, S\left(h_{1}\right)\right)$

for any $c, d \in A, h \in H$.

(5) If the extension is Galois, denote $\operatorname{can}^{-1}\left(1_{A} \otimes h\right)=\sum_{i} l_{i}(h) \otimes_{B} r_{i}(h)$. Then

(5.1) $\sum_{i} l_{i}\left(h_{1}\right) \otimes_{B} r_{i}\left(h_{1}\right) \otimes h_{2}=\sum_{i} l_{i}(h) \otimes_{B} r_{i}(h)_{0} \otimes r_{i}(h)_{1}$.

(5.2) $\sum_{i}^{i} l_{i}(h) r_{i}(h)=\alpha(h) 1_{A}$.

(5.3) $\sum_{i}^{i} l_{i}(h)_{0} \otimes_{B} r_{i}(h) \otimes l_{i}(h)_{1}=\sum_{i} l_{i}\left(h_{2}\right) \otimes_{B} r_{i}\left(h_{2}\right) \otimes S\left(h_{1}\right)$.

(5.4) $\sum_{i}^{i} a_{0} \beta\left(a_{1}\right) l_{i}\left(a_{2}\right) \otimes_{B} r_{i}\left(a_{2}\right)=1_{A} \otimes_{B} a$.

(5.5) $\sum_{i}^{i} l_{i}(h g) \otimes_{B} r_{i}(h g)=\sum_{i, j} \mathbf{f}^{-1}\left(h_{1}, g_{1}\right) l_{i}\left(g_{2}\right) l_{j}\left(h_{2}\right) \otimes_{B} r_{j}\left(h_{2}\right) r_{i}\left(g_{2}\right)$

for all $h, g \in H, a \in A$.

(6) The map can' from Remark 13 is also right $H$-colinear, where the right comodule structure on $A \otimes_{B} A$ is given from the first tensorand, and $A \otimes H$ is a right $H$-comodule via $I_{A} \otimes \Delta$.

Proof. (1) We have

$$
\begin{aligned}
(I \otimes \Delta) \operatorname{can}\left(a \otimes_{B} b\right) & =a_{0} b_{0} \otimes \omega^{-1}\left(a_{1}, b_{1} \beta\left(b_{2}\right), S\left(b_{3}\right)\right) b_{4} \otimes b_{5} \\
& =\operatorname{can}\left(a \otimes_{B} b_{0}\right) \otimes b_{1}
\end{aligned}
$$

(2) Obvious.

(3) Remark first that $\hat{\rho}$ and $\check{\rho}$ are indeed right $H$-comodule structures. Then, for any $a, b \in A$, we compute

$$
\begin{aligned}
\check{\rho} \operatorname{can}\left(a \otimes_{B} b\right) & =\check{\rho}\left(a_{o} b_{0} \otimes \omega^{-1}\left(a_{1}, b_{1} \beta\left(b_{2}\right), S\left(b_{3}\right) b_{4}\right)\right. \\
& =a_{0} b_{0} \otimes b_{6} \otimes\left(a_{1} b_{1}\right) S\left(b_{5}\right) \omega^{-1}\left(a_{2}, b_{2} \beta\left(b_{3}\right), S\left(b_{4}\right)\right) \\
(2.1) & =a_{0} b_{0} \otimes b_{6} \otimes a_{2}\left(b_{2} S\left(b_{4}\right)\right) \omega^{-1}\left(a_{1}, b_{1} \beta\left(b_{3}\right), S\left(b_{5}\right)\right) \\
& =a_{0} b_{0} \otimes b_{4} \otimes a_{2} \omega^{-1}\left(a_{1}, b_{1} \beta\left(b_{2}\right), S\left(b_{3}\right)\right) \\
& =\left(\text { can } \otimes I_{H}\right)\left(a_{0} \otimes_{B} b \otimes a_{1}\right) \\
& =\left(\text { can } \otimes I_{H}\right) \hat{\rho}\left(a \otimes_{B} b\right)
\end{aligned}
$$

(4) We get that

$$
\begin{aligned}
\operatorname{can}\left(c a \otimes_{B} b\right)= & \left(c_{0} a_{0}\right) b_{0} \otimes \omega^{-1}\left(c_{1} a_{1}, b_{1} \beta\left(b_{2}\right), S\left(b_{3}\right)\right) b_{4} \\
= & \left(c_{0} a_{0}\right) b_{0} \otimes \omega^{-1}\left(c_{1}, a_{1}, b_{1}\right) \omega^{-1}\left(c_{2}, a_{2} b_{2}, S\left(b_{6}\right)_{1}\right) \omega^{-1}\left(a_{3}, b_{3}, S\left(b_{6}\right)_{2}\right) \\
& \omega\left(c_{3}, a_{4}, b_{4} S\left(b_{6}\right)_{3}\right) \beta\left(b_{5}\right) b_{7} \\
= & \left(c_{0} a_{0}\right) b_{0} \otimes \omega^{-1}\left(c_{1}, a_{1}, b_{1}\right) \omega^{-1}\left(c_{2}, a_{2} b_{2}, S\left(b_{8}\right)\right) \omega^{-1}\left(a_{3}, b_{3}, S\left(b_{7}\right)\right) \\
& \omega\left(c_{3}, a_{4}, b_{4} \beta\left(b_{5}\right) S\left(b_{6}\right)\right) b_{9} \\
= & \left(c_{0} a_{0}\right) b_{0} \otimes \omega^{-1}\left(c_{1}, a_{1}, b_{1}\right) \omega^{-1}\left(c_{2}, a_{2} b_{2}, S\left(b_{6}\right)\right) \omega^{-1}\left(a_{3}, b_{3}, S\left(b_{5}\right)\right) \beta\left(b_{4}\right) b_{7} \\
= & c_{0}\left(a_{0} b_{0}\right) \otimes \omega^{-1}\left(c_{1}, a_{1} b_{1}, S\left(b_{5}\right)\right) \omega^{-1}\left(a_{2}, b_{2}, S\left(b_{4}\right)\right) \beta\left(b_{3}\right) b_{6}
\end{aligned}
$$

for any $a, b, c \in A$. Now, if we denote $c a n^{-1}(d \otimes h)=\sum_{i} a_{i} \otimes_{B} b_{i}$, then

$$
\left(\rho_{A} \otimes \Delta\right)(d \otimes h)=\sum_{i} a_{i 0} b_{i 0} \otimes a_{i 1} b_{i 1} \otimes \omega^{-1}\left(a_{i 2}, b_{i 2} \beta\left(b_{i 3}\right), S\left(b_{i 4}\right)\right) b_{i 5} \otimes b_{i 6}
$$


Using this, we easily deduce that

$$
\begin{aligned}
\operatorname{can}\left(c \cdot \operatorname{can}^{-1}(d \otimes h)\right) & =\operatorname{can}\left(\sum_{i} c a_{i} \otimes_{B} b_{i}\right) \\
& =c_{0}\left(a_{i 0} b_{i 0}\right) \otimes \omega^{-1}\left(c_{1}, a_{i 1} b_{i 1}, S\left(b_{i 5}\right)\right) \omega^{-1}\left(a_{i 2}, b_{i 2}, S\left(b_{i 4}\right)\right) \beta\left(b_{i 3}\right) b_{i 6} \\
& =c_{0} d_{0} \omega^{-1}\left(c_{1}, d_{1}, S\left(h_{1}\right)\right) \otimes h_{2}
\end{aligned}
$$

(5.1) It follows from (1).

(5.2) We shall check first the formula:

$$
\begin{aligned}
\sum_{i} l_{i}(h) r_{i}(h)= & \left(I_{A} \otimes \varepsilon\right)\left(l_{i}(h)_{0} r_{i}(h)_{0} \otimes \omega\left(l_{i}(h)_{1} r_{i}(h)_{1}, S\left(r_{i}(h)_{6}\right), \alpha\left(r_{i}(h)_{7}\right) r_{i}(h)_{8}\right)\right. \\
& \left.\omega^{-1}\left(l_{i}(h)_{2}, r_{i}(h)_{2} \beta\left(r_{i}(h)_{3}\right), S\left(r_{i}(h)_{4}\right)\right) r_{i}(h)_{5}\right)
\end{aligned}
$$

The left hand side can be also written as:

$$
\begin{aligned}
& \sum_{i}\left(I_{A} \otimes \varepsilon\right)\left(l_{i}(h)_{0} r_{i}(h)_{0} \otimes \omega\left(l_{i}(h)_{1} r_{i}(h)_{1}, S\left(r_{i}(h)_{6}\right), \alpha\left(r_{i}(h)_{7}\right) r_{i}(h)_{8}\right)\right. \\
& \omega^{-1}\left(l_{i}(h)_{2}, r_{i}(h)_{2} \beta\left(r_{i}(h)_{3}\right), S\left(r_{i}(h)_{4}\right)\right) r_{i}(h)_{5} \\
& =\sum_{i} l_{i}(h)_{0} r_{i}(h)_{0} \omega\left(l_{i}(h)_{1} r_{i}(h)_{1}, S\left(r_{i}(h)_{5}\right), \alpha\left(r_{i}(h)_{6}\right) r_{i}(h)_{7}\right) \\
& \omega^{-1}\left(l_{i}(h)_{2}, r_{i}(h)_{2} \beta\left(r_{i}(h)_{3}\right), S\left(r_{i}(h)_{4}\right)\right) \\
(\underline{2.3}) & =\sum_{i} l_{i}(h)_{0} r_{i}(h)_{0} \omega^{-1}\left(l_{i}(h)_{1}, r_{i}(h)_{1}, S\left(r_{i}(h)_{5}\right)_{1} r_{i}(h)_{7_{1}}\right) \omega\left(r_{i}(h)_{2}, S\left(r_{i}(h)_{5}\right)_{2}, r_{i}(h)_{7_{2}}\right) \\
& \alpha\left(r_{i}(h)_{6}\right) \beta\left(r_{i}(h)_{4}\right) \omega\left(l_{i}(h)_{2}, r_{i}(h)_{3} S\left(r_{i}(h)_{5}\right), r_{i}(h)_{7_{3}}\right) \\
& =\sum_{i} l_{i}(h) r_{i}(h)_{0} \omega\left(r_{i}(h)_{1}, S\left(r_{i}(h)_{3}\right), r_{i}(h)_{5}\right) \alpha\left(r_{i}(h)_{4}\right) \beta\left(r_{i}(h)_{2}\right) \\
& =\sum_{i} l_{i}(h) r_{i}(h)_{0} \varepsilon\left(r_{i}(h)_{1}\right) \\
& =\sum_{i} l_{i}(h) r_{i}(h)
\end{aligned}
$$

But $\sum_{i} l_{i}(h)_{0} r_{i}(h)_{0} \otimes \omega^{-1}\left(l_{i}(h)_{1}, r_{i}(h)_{1} \beta\left(r_{i}(h)_{2}\right), S\left(r_{i}(h)_{3}\right)\right) r_{i}(h)_{4}=1_{A} \otimes h$, therefore

$$
\begin{aligned}
\sum_{i} l_{i}(h) r_{i}(h) & =\left(I_{A} \otimes \varepsilon\right)\left(1_{A} \otimes h_{1} \omega\left(1_{H}, S\left(h_{2}\right), \alpha\left(h_{3}\right) h_{4}\right)\right) \\
& =1_{A} \alpha(h)
\end{aligned}
$$

(5.3) It results from (3).

(5.4) We compute

$$
\begin{aligned}
\operatorname{can}\left(\sum_{i} a_{0} \beta\left(a_{1}\right) l_{i}\left(a_{2}\right) \otimes_{B} r_{i}\left(a_{2}\right)\right)= & {\left[a_{0} \beta\left(a_{2}\right) l_{i}\left(a_{3}\right)_{0}\right] r_{i}\left(a_{3}\right)_{0} \otimes r_{i}\left(a_{3}\right)_{4} } \\
& \omega^{-1}\left(a_{1} l_{i}\left(a_{3}\right)_{1}, r_{i}\left(a_{3}\right)_{1} \beta\left(r_{i}\left(a_{3}\right)_{2}\right), S\left(r_{i}\left(a_{3}\right)_{3}\right)\right. \\
= & a_{0}\left[l_{i}\left(a_{4}\right)_{0} r_{i}\left(a_{4}\right)_{0}\right] \beta\left(a_{3}\right) \omega\left(a_{1}, l_{i}\left(a_{4}\right)_{1}, r_{i}\left(a_{4}\right)_{1}\right) \otimes r_{i}\left(a_{4}\right)_{5} \\
& \omega^{-1}\left(a_{2} l_{i}\left(a_{4}\right)_{2}, r_{i}\left(a_{4}\right)_{2} \beta\left(r_{i}\left(a_{4}\right)_{3}\right), S\left(r_{i}\left(a_{4}\right)_{4}\right)\right. \\
= & a_{0}\left[l_{i}\left(a_{4}\right)_{0} r_{i}\left(a_{4}\right)_{0}\right] \beta\left(a_{3}\right) \otimes r_{i}\left(a_{4}\right)_{8}
\end{aligned}
$$




$$
\begin{aligned}
& \omega^{-1}\left(a_{1}, l_{i}\left(a_{4}\right)_{1} r_{i}\left(a_{4}\right)_{1}, S\left(r_{i}\left(a_{4}\right)_{7}\right)\right) \beta\left(r_{i}\left(a_{4}\right)_{4}\right) \\
& \omega^{-1}\left(l_{i}\left(a_{4}\right)_{2}, r_{i}\left(a_{4}\right)_{2}, S\left(r_{i}\left(a_{4}\right)_{6}\right)\right) \\
& \omega\left(a_{2}, l_{i}\left(a_{4}\right)_{3}, r_{i}\left(a_{4}\right)_{3} S\left(r_{i}\left(a_{4}\right)_{5}\right)\right. \\
= & a_{0}\left[l_{i}\left(a_{3}\right)_{0} r_{i}\left(a_{3}\right)_{0}\right] \beta\left(a_{2}\right) \otimes r_{i}\left(a_{3}\right)_{6} \\
& \omega^{-1}\left(a_{1}, l_{i}\left(a_{3}\right)_{1} r_{i}\left(a_{3}\right)_{1}, S\left(r_{i}\left(a_{3}\right)_{5}\right)\right) \\
& \omega^{-1}\left(l_{i}\left(a_{3}\right)_{2}, r_{i}\left(a_{3}\right)_{2}, S\left(r_{i}\left(a_{3}\right)_{4}\right)\right) \beta\left(r_{i}\left(a_{3}\right)_{3}\right) \\
= & a_{0} \beta\left(a_{2}\right) 1_{A} \otimes a_{4} \omega^{-1}\left(a_{1}, 1_{H}, S\left(a_{3}\right)\right) \\
= & a_{0} \beta\left(a_{1}\right) \otimes a_{2} \\
= & \operatorname{can}\left(1_{A} \otimes_{B} a\right)
\end{aligned}
$$

(5.5) It is a consequence of the previous properties of $c a n$ and of the properties (2.15)-(2.22) of the twist f.

(6) Easy.

Remark 24. As a consequence of Proposition 231(3), and 23(4), we obtain that can is a morphism of left Hopf modules, where $A^{\bullet} \otimes_{B} A$ is an object in ${ }_{A} \mathcal{M}^{H}$ with structures given by the first tensorand, while - $A^{\bullet} \otimes H^{S, \bullet}$ is the induced module in ${ }_{A} \mathcal{M}^{H}$. Here $H^{S, \bullet}$ is the right comodule structure of $H$ deformed by the antipode $S$ (i.e. $\bullet A^{\bullet} \otimes H^{S, \bullet}$ is a left Hopf module with structure morphisms $a \otimes h \longrightarrow a_{0} \otimes h_{2} \otimes a_{1} S\left(h_{1}\right)$, $a(b \otimes h)=a_{0} b_{0} \otimes h_{2} \omega^{-1}\left(a_{1}, b_{1}, S\left(h_{1}\right)\right)$.

Definition 25. Let $A$ a right $H$-comodule algebra and $\gamma: H \longrightarrow A$ a colinear map. The extension $B \subseteq A$ is $(H, S)$-cleft with respect to the cleaving map $\gamma$ if there is a linear map $\delta_{\gamma, S}: H \longrightarrow A$ such that

$$
\begin{aligned}
\rho\left(\delta_{\gamma, S}(h)\right) & =\delta_{\gamma, S}\left(h_{2}\right) \otimes S\left(h_{1}\right) \\
\delta_{\gamma, S}\left(h_{1}\right) \gamma\left(h_{2}\right) & =\alpha(h) 1_{A} \\
\gamma\left(h_{1}\right) \beta\left(h_{2}\right) \delta_{\gamma, S}\left(h_{3}\right) & =\varepsilon(h) 1_{A}
\end{aligned}
$$

Remark 26. (1) This definition of cleftness is slightly different from the classical one. In the Hopf case, it is only required that $\gamma$ is convolution invertible (denote by $\delta$ the convolution inverse of $\gamma$ ) and $H$-colinear. The property (3.7) appears naturally by passing from a bialgebra to a Hopf algebra. Unfortunately, in our case the convolution product on $\operatorname{Hom}(H, A)$ is no longer associative, therefore a left inverse for $\gamma$ is not necessarily a right inverse and the property (3.7) does not seem to result from the other properties of $\gamma$. So we had to state it separately.

(2) For a cleft comodule algebra $A$, the application $\delta_{\gamma, S}$ depends on the antipode. If we change it to $\left(S^{\prime}, \alpha^{\prime}, \beta^{\prime}\right)$ as in (2.8) and define $\delta_{\gamma, S^{\prime}}(h)=U\left(h_{1}\right) \delta_{\gamma, S}\left(h_{2}\right)$, then it follows immediately that $A$ is also $\left(H, S^{\prime}\right)$ cleft. In the sequel, we shall drop the subscripts for simplicity, considering the antipode and the elements $\alpha$, $\beta$ fixed once for all.

Recall that the "normal basis property" states that there is an isomorphism of left $B$-modules, right $H$ comodules $A \simeq \boldsymbol{\bullet} B \otimes H^{\bullet}$, where the dots are indicating the corresponding structures for the tensor product. We shall keep the same definition for coquasi-Hopf algebras, as nothing is changed.

Theorem 27. Let $H$ be a coquasi-Hopf algebra with bijective antipode, $A$ a right $H$-comodule algebra with $B$ the subalgebra of coinvariants. Then the following statements are equivalent:

(1) The extension $B \subseteq A$ is $H$-cleft;

(2) The Weak Structure Theorem holds and the extension has the normal basis property;

(3) The extension $B \subseteq A$ is $H$-Galois and has the normal basis property.

In this case, the categories $\mathcal{M}_{B}$ and $\mathcal{M}_{A}^{H}$ are equivalent (the Strong Structure Theorem holds). 
Proof. The proof of this theorem follows closely the original one for Hopf algebras, due to Doi and Takeuchi ([17]), and Blattner and Montgomery ([7]), but we shall do the computations in detail, because of their degree of difficulty.

$(1) \Longrightarrow(2)$ Define

$$
\nu: B \otimes H \longrightarrow A, \quad \nu(b \otimes h)=b \gamma(h)
$$

It is obvious $B$-linear. As $\gamma$ is $H$-colinear, $\nu$ will also be. We need an inverse for $\nu$. We take

$$
\nu^{-1}(a)=a_{0} \delta\left(a_{1}<\beta\right) \otimes a_{2}, \quad a \in A
$$

We need to show first that it is well-defined. For all $a \in A$, we have

$$
\begin{aligned}
\rho_{A}\left(a _ { 0 } \delta \left(a_{1}\right.\right. & <\beta))=a_{0_{0}} \delta\left(a_{1} \leftarrow \beta\right)_{0} \otimes a_{0_{1}} \delta\left(a_{1} \leftarrow \beta\right)_{1} \\
\text { (3.7) } & =a_{0} \delta\left(a_{4}\right) \otimes a_{1} \beta\left(a_{2}\right) S\left(a_{3}\right) \\
& =a_{0} \beta\left(a_{1}\right) \delta\left(a_{2}\right) \otimes 1_{H} \\
& =a_{0} \delta\left(a_{1} \leftarrow \beta\right) \otimes 1_{H}
\end{aligned}
$$

meaning that $\operatorname{Im} \nu^{-1} \subseteq B \otimes H$. Let's check now that $\nu$ and $\nu^{-1}$ are indeed inverses to each other: for all $a \in A$, we compute

$$
\begin{aligned}
\left(\nu \circ \nu^{-1}\right)(a) & =\nu\left(a_{0} \delta\left(a_{1} \leftarrow \beta\right) \otimes a_{2}\right) \\
& =\left[a_{0} \delta\left(a_{1} \leftarrow \beta\right)\right] \gamma\left(a_{2}\right) \\
& =a_{0}\left[\delta\left(a_{2} \leftarrow \beta\right)_{0} \gamma\left(a_{3}\right)_{0}\right] \omega\left(a_{1}, \delta\left(a_{2} \leftarrow \beta\right)_{1}, \gamma\left(a_{3}\right)_{1}\right) \\
& =a_{0}\left[\delta\left(a_{4}\right) \gamma\left(a_{5}\right)\right] \omega\left(a_{1}, S\left(a_{3}\right), a_{6}\right) \beta\left(a_{2}\right) \\
& =a_{0} \omega\left(a_{1}, S\left(a_{3}\right), a_{5}\right) \beta\left(a_{2}\right) \alpha\left(a_{4}\right) \\
& =a
\end{aligned}
$$

Conversely, for $b \in B$ and $h \in H$ we get

$$
\begin{aligned}
\nu^{-1} \circ \nu(b \otimes h) & =\nu^{-1}(b \gamma(h)) \\
& =b \gamma(h)_{0} \delta\left(\left(\gamma(h)_{1}\right) \leftarrow \beta\right) \otimes \gamma(h)_{2} \\
& =b \gamma\left(h_{1}\right) \beta\left(h_{2}\right) \delta\left(h_{3}\right) \otimes h_{4} \\
& =b \otimes h
\end{aligned}
$$

Hence the extension $B \subseteq A$ has the normal basis property. It remains only to show the bijectivity of the adjunction counit from Proposition 7 . For a Hopf module $M \in \mathcal{M}_{A}^{H}$, define the map $t_{M}: M \longrightarrow M$ by $t_{M}(m)=m_{0} \delta\left(m_{1} \leftarrow \beta\right)$. Then we can see as in (3.12) that the image of $t_{M}$ is in $M^{c o H}$. Define now $\chi: M \longrightarrow M^{c o H} \otimes_{B} A, \chi(m)=t_{M}\left(m_{0}\right) \otimes_{B} \gamma\left(m_{1}\right)$. Computing as in (3.13), we get that $\chi$ is an inverse for $\varepsilon_{M}$.

(2) $\Longrightarrow(3)$ It follows from Corollary (21).

(3) $\Longrightarrow(1)$ Let $\nu: B \otimes H \longrightarrow A$ be the isomorphism given by the normal basis property. Define $\gamma(h)=\nu\left(1_{A} \otimes h\right)$. As $\nu$ is $H$-colinear, $\gamma$ will also be.

In order to get the second map $\delta$, we need some work first. Consider the map $\Gamma=\left(I_{A} \otimes \varepsilon\right) \nu^{-1}: A \longrightarrow B$. Then $\Gamma$ is left $B$-linear, as $\nu^{-1}$ is $B$-linear, and

$$
\begin{aligned}
\Gamma \gamma(h) & =\left(I_{A} \otimes \varepsilon\right) \nu^{-1} \nu\left(1_{A} \otimes h\right) \\
& =\varepsilon(h) 1_{A}
\end{aligned}
$$


Now we may take $\delta(h)=m_{A}\left(I_{A} \otimes_{B} \Gamma\right) c a n^{-1}(1 \otimes h)=\sum_{i} l_{i}(h) \Gamma\left(r_{i}(h)\right)$, where $m_{A}$ is the multiplication on $A$. We may then compute

$$
\begin{aligned}
\gamma\left(h_{1}\right) \beta\left(h_{2}\right) \delta\left(h_{3}\right) & =\underbrace{\gamma\left(h_{1}\right)}_{\in A} m_{A}\left(I_{A} \otimes_{B} \Gamma\right) \operatorname{can}^{-1}\left(1_{A} \otimes \beta\left(h_{2}\right) h_{3}\right) \\
& =m_{A}\left(I_{A} \otimes_{B} \Gamma\right)\left[\gamma\left(h_{1}\right) \operatorname{can}^{-1}\left(1_{A} \otimes \beta\left(h_{2}\right) h_{3}\right)\right] \\
\text { (Proposition 23) } & =m_{A}\left(I_{A} \otimes_{B} \Gamma\right) \operatorname{can}^{-1}\left(\gamma\left(h_{1}\right)_{0} \otimes \beta\left(h_{2}\right) h_{4}\right) \omega^{-1}\left(\gamma\left(h_{1}\right)_{1}, 1, S\left(h_{3}\right)\right) \\
& =m_{A}\left(I_{A} \otimes_{B} \Gamma\right) \operatorname{can}^{-1}\left(\gamma\left(h_{1}\right) \otimes \beta\left(h_{2}\right) h_{3}\right) \\
(\gamma \text { is colinear) } & =m_{A}\left(I_{A} \otimes_{B} \Gamma\right) \operatorname{can}^{-1}\left(\gamma(h)_{0} \otimes \beta\left(\gamma(h)_{1}\right) \gamma(h)_{2}\right) \\
(\text { Proposition 23) } & =m_{A}\left(I_{A} \otimes_{B} \Gamma\right)\left(1_{A} \otimes_{B} \gamma(h)\right) \\
& =\Gamma \gamma(h) \\
& =\varepsilon(h) 1_{A}
\end{aligned}
$$

For the last formula, notice first that $H$-colinearity of $\nu$ implies

$$
\begin{aligned}
\nu^{-1} & =\left(I_{B} \otimes \varepsilon \otimes I_{H}\right)\left(I_{B} \otimes \Delta\right) \nu^{-1} \\
& =\left(I_{B} \otimes \varepsilon \otimes I_{H}\right)\left(\nu^{-1} \otimes I_{H}\right) \rho_{A} \\
& =\left(\Gamma \otimes I_{H}\right) \rho_{A}
\end{aligned}
$$

Now we may compute

$$
\begin{aligned}
\delta\left(h_{1}\right) \gamma\left(h_{2}\right) & =\left[m_{A}\left(I_{A} \otimes \otimes_{B} \Gamma\right) \operatorname{can}^{-1}\left(1_{A} \otimes h_{1}\right)\right] \nu\left(1_{A} \otimes h_{2}\right) \\
& =\sum_{i}[l_{i}\left(h_{1}\right) \underbrace{\Gamma\left(r_{i}\left(h_{1}\right)\right)}_{\in B}] \nu\left(1_{A} \otimes h_{2}\right) \\
\text { Proposition 23) } & =\sum_{i}[l_{i}(h) \underbrace{\Gamma\left(r_{i}(h)_{0}\right)}_{\in B}] \nu\left(1_{A} \otimes r_{i}(h)_{1}\right) \\
& =\sum_{i} l_{i}(h)\left[\Gamma\left(r_{i}(h)_{0}\right) \nu\left(1_{A} \otimes r_{i}(h)_{1}\right)\right] \\
& =\sum_{i} l_{i}(h) \nu\left(\Gamma\left(r_{i}(h)_{0} \otimes r_{i}(h)_{1}\right)\right. \\
& =\sum_{i} l_{i}(h) r_{i}(h) \\
(\text { Proposition 23) } & =\alpha(h) 1_{A}
\end{aligned}
$$

for all $h \in H$.

Finally, for any $h \in H$ we have

$$
\begin{aligned}
\rho_{A} \delta(h) & =\rho_{A} m_{A}\left(I_{A} \otimes_{B} \Gamma\right) \operatorname{can}^{-1}\left(1_{A} \otimes h\right) \\
& =\rho_{A}\left(\sum_{i} l_{i}(h) \Gamma\left(r_{i}(h)\right)\right) \\
& =\sum_{i} l_{i}(h)_{0} \Gamma\left(r_{i}(h)\right) \otimes l_{i}(h)_{1} \\
(\text { Proposition 23) }) & =\sum_{i} l_{i}\left(h_{2}\right) \Gamma\left(r_{i}\left(h_{2}\right)\right) \otimes S\left(h_{1}\right) \\
& =\delta\left(h_{2}\right) \otimes S\left(h_{1}\right)
\end{aligned}
$$


For the remaining of the theorem, the proof is the same as in [17, so we omit it.

We shall prove now an imprimitivity statement which is the analogue of Doi's and Takeuchi's theorem ([18]) and Schneider's theorem ([33]) for coquasi-Hopf algebras.

Theorem 28. Let $H$ be a coquasi-Hopf algebra with bijective antipode, $A$ a right $H$-comodule algebra with $B$ the algebra of coinvariants. Then the following are equivalent:

(1) $A$ is faithfully flat as a left B-module, and $A$ is a Galois extension of B.

(2) The functor of coinvariants and the induction functor are a pair of inverse equivalences between $\mathcal{M}_{A}^{H}$ and $\mathcal{M}_{B}$.

Proof. (1) $\Longrightarrow(2)$ We need first a Lemma:

Lemma 29. Let $H$ be a coquasi-Hopf algebra and $A$ a right comodule algebra. Then the Galois map can induces a natural right colinear morphism can c $_{M}: M \otimes_{B} A^{\bullet} \longrightarrow M \otimes H^{\bullet}, \operatorname{can}_{M}\left(m \otimes_{B} a\right)=m_{0} a_{0} \otimes$ $\omega^{-1}\left(m_{1}, a_{1} \beta\left(a_{2}\right), S\left(a_{3}\right)\right) a_{4}$. If can is bijective, then can ${ }_{M}$ is also bijective.

Proof. (of the Lemma) The definition of $\operatorname{can}_{M}$ allows us to easily check its colinearity. The hard part is the proof of the naturality and of the bijectivity of $\operatorname{can}_{M}$. This is not obvious at all, because $A$ is no longer an associative algebra and the classical argument (i.e. tensoring over $A$ ) is not working. In this case it is more appropriate to work in the monoidal category of comodules. We refer to 4 for details about tensor product over an algebra in a monoidal category. As $A$ is an algebra in the monoidal abelian category $\mathcal{M}^{H}$, we may form the tensor product $M \bigcirc_{A} M^{\prime}$ for any $M \in \mathcal{M}_{A}^{H}, M^{\prime} \in{ }_{A} \mathcal{M}^{H}$ as the following equalizer

$$
(M \otimes A) \otimes M^{\prime} \underset{\left(I_{M} \otimes \mu_{M^{\prime}}\right) \phi_{M, A, M^{\prime}}}{\stackrel{\mu_{M} \otimes I_{M^{\prime}}}{\rightrightarrows}} M \otimes M^{\prime} \longrightarrow M \bigcirc_{A} M^{\prime} \longrightarrow 0
$$

where $\mu_{M}$ and $\mu_{M^{\prime}}$ are the $A$-module structure maps and $\phi$ is the coassociator.

We need now two particular left Hopf modules. One of them is $\bullet^{\bullet} \otimes_{B} A$, with right $H$-coaction and left $A$-action on the first component. For the other one, notice first that $S$ is a coalgebra map. Therefore we may take $H$ as an object in $\mathcal{M}^{H}$ with $h \longrightarrow h_{2} \otimes S\left(h_{1}\right)$, denoted $H^{S}$. Then we get an induced left Hopf module $\bullet A^{\bullet} \otimes H^{S, \bullet} \in{ }_{A} \mathcal{M}^{H}$, with structure maps

$$
\begin{aligned}
a \otimes h & \longrightarrow a_{0} \otimes h_{2} \otimes a_{1} S\left(h_{1}\right) \\
a(b \otimes h) & =a_{0} b_{0} \otimes h_{2} \omega^{-1}\left(a_{1}, b_{1}, S\left(h_{1}\right)\right)
\end{aligned}
$$

We can construct now the following diagram for any $M \in \mathcal{M}_{A}^{H}$ :

$$
\begin{aligned}
& \begin{array}{rr} 
& M \otimes_{B} A \\
& F_{1} \nearrow \swarrow G_{1} \quad \widetilde{F}_{1} \uparrow \downarrow \widetilde{F}_{1}^{-1}
\end{array} \\
& (M \otimes A) \otimes\left(A \otimes_{B} A\right) \\
& \mu_{M} \otimes\left(I_{A} \otimes_{B} I_{A}\right) \\
& \left(I_{M} \otimes\left(m_{A} \otimes_{B} I_{A}\right) \phi_{M, A, A \otimes_{B} A}\right. \\
& M \otimes\left(A \otimes_{B} A\right) \\
& \stackrel{\pi_{1}}{\longrightarrow} \\
& M \bigcirc_{A}\left(A \otimes_{B} A\right) \longrightarrow 0 \\
& \downarrow_{I_{M} \otimes c a n} \\
& (M \otimes A) \otimes(A \otimes H)
\end{aligned}
$$

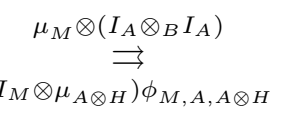

$$
\begin{aligned}
& M \otimes(A \otimes H) \\
& \downarrow_{I_{M} \bigcirc_{A} \text { can }} \\
& \stackrel{\pi_{2}}{\longrightarrow} \\
& M \bigcirc_{A}(A \otimes H) \longrightarrow 0 \\
& F_{2} \searrow \nwarrow G_{2} \quad \widetilde{F}_{2} \downarrow \uparrow \widetilde{G}_{2} \\
& M \otimes H
\end{aligned}
$$

The two rows are exact by definition of $\bigcirc_{A}$. The application $F_{1}: M \bullet \otimes\left(\bullet A^{\bullet} \otimes_{B} A\right) \longrightarrow M \bullet \otimes_{B} A$, $F_{1}\left(m \otimes\left(a \otimes_{B} b\right)\right)=m a \otimes_{B} b$ is well-defined, right $H$-colinear and

$$
F_{1}\left(\mu_{M} \otimes\left(I_{A} \otimes_{B} I_{A}\right)\right)=F_{1}\left(I_{M} \otimes\left(m_{A} \otimes_{B} I_{A}\right)\right) \phi_{M, A, A \otimes_{B} A}
$$


(here $M^{\bullet} \otimes_{B} A$ is a right comodule via $\rho_{M} \otimes_{B} I_{A}$, while $M_{\bullet}^{\bullet} \otimes\left(\bullet A^{\bullet} \otimes_{B} A\right)$ has the codiagonal comodule structure). Hence there is a right $H$-comodule morphism $\widetilde{F}_{1}: M \bigcirc_{A}\left(A \otimes_{B} A\right) \longrightarrow M \otimes_{B} A$ such that $\widetilde{F}_{1} \pi_{1}=F_{1}$. Moreover, $F_{1}$ is an isomorphism with inverse $\widetilde{F}_{1}^{-1}=\pi_{1} G_{1}$, where $G_{1}\left(m \otimes_{B} a\right)=m \otimes\left(1_{A} \otimes_{B} a\right)$.

As $\bullet A^{\bullet} \otimes H^{S, \bullet}$ is an $A$-module induced in $\mathcal{M}^{H}$, the colinear map $F_{2}: M_{\bullet} \otimes\left(\bullet A^{\bullet} \otimes H^{S, \bullet}\right) \longrightarrow M^{\bullet} \otimes H^{S, \bullet}$, $F_{2}(m \otimes(a \otimes h))=m_{0} a_{0} \otimes h_{2} \omega^{-1}\left(m_{1}, a_{1}, S\left(h_{1}\right)\right)$ factors through an isomorphism of right $H$-comodules $\widetilde{F}_{2}: M \bigcirc_{A}(A \otimes H) \simeq M \otimes H$, with inverse $\widetilde{F}_{2}^{-1}=\pi_{2} G_{2}$, where $G_{2}(m \otimes h)=m \otimes\left(1_{A} \otimes h\right)\left(\right.$ on $M^{\bullet} \otimes H^{S, \bullet}$ we have again the codiagonal comodule structure).

According to Remark 24, the map can : $A^{\bullet} \otimes_{B} A \longrightarrow \bullet A^{\bullet} \otimes H^{S, \bullet}$ is a morphism in ${ }_{A} \mathcal{M}^{H}$. Then $I_{M} \otimes c a n$ induces a colinear map $I_{M} \bigcirc_{A}$ can $: M \bigcirc_{A}\left(A \otimes_{B} A\right) \longrightarrow M \bigcirc_{A}\left(A \otimes_{B} A\right)$.

Composing now the morphisms from the last column in the above diagram, we obtain a natural map $\operatorname{can}_{M}: M \otimes_{B} A \longrightarrow M \otimes H$, which can be written as $\operatorname{can}_{M}=\widetilde{F}_{2}\left(I_{M} \bigcirc_{A}\right.$ can $) \widetilde{F}_{1}^{-1}=F_{2}\left(I_{M} \otimes c a n\right) G_{1}$. This implies

$$
\begin{aligned}
\operatorname{can}_{M}\left(m \otimes_{B} a\right) & =F_{2}\left(I_{M} \otimes \operatorname{can}\right) G_{1}\left(m \otimes_{B} a\right) \\
& =F_{2}\left(I_{M} \otimes \operatorname{can}\right)\left(m \otimes\left(1_{A} \otimes_{B} a\right)\right) \\
& =F_{2}\left(m \otimes\left(a_{0} \otimes \beta\left(a_{1}\right) a_{2}\right)\right) \\
& =m_{0} a_{0} \otimes \omega^{-1}\left(m_{1}, a_{1} \beta\left(a_{2}\right), S\left(a_{3}\right)\right) a_{4}
\end{aligned}
$$

The last part of the Lemma is now obvious.

(Proof of the Theorem) As in Lemma 19, we are able to show that for any $M \in \mathcal{M}_{A}^{H}, M \otimes H$ becomes an object in $\mathcal{M}_{A}^{H}$, with structure morphisms as in Remark 20 (replacing the elements of $A$ with elements of $M)$. For later use, we write down explicitly the used isomorphisms:

$$
\begin{gathered}
H^{\bullet} \otimes M \bullet \underset{\eta_{M}^{-1}}{\stackrel{\eta_{M}}{\rightleftarrows}} M \otimes H^{\bullet}, \\
\eta_{M}(h \otimes m)=m_{0} \otimes \omega\left(h_{1}, m_{3}, \alpha S^{-1}\left(m_{2}\right) S^{-1}\left(m_{1}\right)\right) h_{2} m_{4} \\
\eta_{M}^{-1}(m \otimes h)=h_{1} S^{-1}\left(m_{4}\right) \otimes m_{0} \omega^{-1}\left(h_{2}, S^{-1}\left(m_{3}\right) \beta S^{-1}\left(m_{2}\right), m_{1}\right)
\end{gathered}
$$

It follows that $\varepsilon_{M \otimes H}=\left(\operatorname{can}_{M}\right)_{\tilde{\mathbf{f}}}$ is bijective. Repeating the argument with $M \otimes H$ instead of $M$, we obtain the bijectivity of $\varepsilon_{(M \otimes H) \otimes H}=\left(\operatorname{can}_{M \otimes H}\right)_{\tilde{\mathbf{f}}}$. Now the trick is to put this three maps together in a commutative diagram using their naturality:

$$
\begin{array}{cccccc}
0 \longrightarrow & M^{c o H} \otimes_{B} A & \longrightarrow & M \otimes_{B} A & \rightrightarrows & \left((M \otimes H) \otimes_{B} A\right. \\
\varepsilon_{M} \downarrow & & \varepsilon_{M \otimes H} \downarrow & & \varepsilon_{(M \otimes H) \otimes H \downarrow} \\
0 \longrightarrow & M & \stackrel{\widetilde{\rho}_{M}}{\longrightarrow} & M \otimes H & \stackrel{I_{M} \otimes \widetilde{\Delta}}{\rightrightarrows} & (M \otimes H) \otimes H
\end{array}
$$

On the bottom row, $\widetilde{\rho}_{M}(m)=m_{0} \otimes \alpha S^{-1}\left(m_{1}\right) m_{2}$ and $\widetilde{\Delta}(h)=h_{1} \otimes \alpha S^{-1}\left(h_{2}\right) h_{3}$ for $m \in M, h \in H$. The upper row contains their images via the functor $(-)^{c o H} \otimes_{B} A$. We need to see that $\widetilde{\rho}_{M}, \widetilde{\rho}_{M} \otimes I_{H}$ and $I_{M} \otimes \widetilde{\Delta}$ are morphisms of Hopf modules and the bottom row is exact. It is easy to check the colinearity of these maps, as the comodule structure on the tensor products $M \otimes H$ and $M \otimes H \otimes H$ comes from the last tensorand. The difficult part is the right $A$-linearity, because of the unpleasant formula (3.5) (applied to $M$, respectively $M \otimes H$ ). Instead of checking it directly, we shall use the isomorphism which leads to the mentioned formula. For $\widetilde{\rho}_{M}$, we have:

$$
M \stackrel{\widetilde{\rho}_{M}}{\longrightarrow} M \otimes H \stackrel{\eta_{M}^{-1}}{\simeq} H \otimes M
$$


and

$$
\begin{aligned}
m & \longrightarrow m_{0} \otimes \alpha S^{-1}\left(m_{1}\right) m_{2} \stackrel{\eta_{M}^{-1}}{\longrightarrow} m_{6} \alpha S^{-1}\left(m_{5}\right) S^{-1}\left(m_{4}\right) \otimes m_{0} \omega^{-1}\left(m_{7}, S^{-1}\left(m_{3}\right) \beta S^{-1}\left(m_{2}\right), m_{1}\right) \\
(2.5) & =\alpha S^{-1}\left(m_{4}\right) 1_{H} \otimes m_{0} \omega^{-1}\left(m_{5}, S^{-1}\left(m_{3}\right) \beta S^{-1}\left(m_{2}\right), m_{1}\right) \\
(2.7) & =1_{H} \otimes m
\end{aligned}
$$

Remembering that the $A$-module structure of $H \otimes M$ is the one induced by $M$ (i.e. $(h \otimes m) a=h_{1} \otimes$ $\left.m_{0} a_{0} \omega\left(h_{2}, m_{1}, a_{1}\right)\right)$, it is now easy to verify the $A$-linearity for the composed map $\eta_{M}^{-1} \widetilde{\rho}_{M}$.

For $\widetilde{\rho}_{M} \otimes I_{H}$, we need to compose three times with the following isomorphisms

$$
H^{\bullet} \otimes M_{\bullet} \stackrel{\eta_{M}}{\simeq} M_{\bullet} \otimes H_{\bullet} \stackrel{\widetilde{\rho}_{M} \otimes I_{H}}{\longrightarrow}\left(M_{\bullet} \otimes H_{\bullet}\right) \bullet \otimes H_{\bullet} \stackrel{\eta_{M \otimes H}}{\simeq} H^{\bullet} \otimes\left(M_{\bullet} \otimes H_{\bullet}\right): \stackrel{I_{H} \otimes \eta_{M}^{-1}}{\simeq} H^{\bullet} \otimes\left(H^{\bullet} \otimes M_{\bullet}\right):
$$

We use dots to indicate the structures (although the right $A$-structures are not the classical ones, we find the notation more suggestive). We obtain

$$
\begin{aligned}
h \otimes m \longrightarrow & m_{0} \otimes \omega\left(h_{1}, m_{3}, \alpha S^{-1}\left(m_{2}\right) S^{-1}\left(m_{1}\right)\right) h_{2} m_{4} \\
\longrightarrow & m_{0} \otimes \alpha S^{-1}\left(m_{1}\right) m_{2} \otimes \omega\left(h_{1}, m_{5}, \alpha S^{-1}\left(m_{4}\right) S^{-1}\left(m_{3}\right)\right) h_{2} m_{6} \\
\longrightarrow & h_{1} \otimes m_{0} \otimes \alpha S^{-1}\left(m_{1}\right) m_{2} \omega^{-1}\left(h_{3} m_{9}, S^{-1}\left(m_{5}\right) \beta S^{-1}\left(m_{4}\right), m_{3}\right) \omega\left(h_{2}, m_{8} \alpha S^{-1}\left(m_{7}\right), S^{-1}\left(m_{6}\right)\right) \\
= & h_{1} \otimes m_{0} \otimes \alpha S^{-1}\left(m_{1}\right) m_{2} \omega^{-1}\left(h_{2}, m_{11} S^{-1}\left(m_{9}\right), m_{3}\right) \omega^{-1}\left(m_{12}, S^{-1}\left(m_{8}\right), m_{4}\right) \\
& \omega\left(h_{3}, m_{10}, S^{-1}\left(m_{7}\right) m_{5}\right) \beta S^{-1}\left(m_{6}\right) \alpha S^{-1}\left(m_{10}\right) \\
= & h \otimes m_{0} \otimes \alpha S^{-1}\left(m_{1}\right) m_{2} \omega^{-1}\left(m_{7}, S^{-1}\left(m_{5}\right), m_{3}\right) \beta S^{-1}\left(m_{4}\right) \alpha S^{-1}\left(m_{6}\right) \\
\text { (2.3) } & h \otimes m_{0} \otimes \alpha S^{-1}\left(m_{1}\right) m_{2} \\
& \text { (2.6) }
\end{aligned}
$$

and this is again right $A$-linear.

Finally, we repeat the above composition with $I_{M} \otimes \widetilde{\Delta}$ instead of $\widetilde{\rho}_{M} \otimes I_{H}$. We obtain

$$
\begin{aligned}
h \otimes m \longrightarrow & m_{0} \otimes \omega\left(h_{1}, m_{3}, \alpha S^{-1}\left(m_{2}\right) S^{-1}\left(m_{1}\right)\right) h_{2} m_{4} \\
\longrightarrow & m_{0} \otimes \omega\left(h_{1}, m_{3}, \alpha S^{-1}\left(m_{2}\right) S^{-1}\left(m_{1}\right)\right) h_{2} m_{4} \otimes \alpha S^{-1}\left(h_{3} m_{5}\right) h_{4} m_{6} \\
\longrightarrow & h_{8} m_{10} \alpha S^{-1}\left(h_{7} m_{9}\right) S^{-1}\left(h_{6} m_{8}\right) \otimes m_{0} \otimes \omega\left(h_{1}, m_{3}, \alpha S^{-1}\left(m_{2}\right) S^{-1}\left(m_{1}\right)\right) h_{2} m_{4} \\
& \omega^{-1}\left(h_{9} m_{11}, S^{-1}\left(h_{5} m_{7}\right) \beta S^{-1}\left(h_{4} m_{6}\right), h_{3} m_{5}\right) \\
= & 1_{H} \otimes m_{0} \otimes \omega\left(h_{1}, m_{3}, \alpha S^{-1}\left(m_{2}\right) S^{-1}\left(m_{1}\right)\right) h_{2} m_{4} \omega^{-1}\left(h_{7} m_{9}, S^{-1}\left(h_{5} m_{7}\right)\right. \\
& \left.\beta S^{-1}\left(h_{4} m_{6}\right), h_{3} m_{5}\right) \alpha S^{-1}\left(h_{6} m_{8}\right) \\
& =1_{H} \otimes m_{0} \otimes \omega\left(h_{1}, m_{3}, \alpha S^{-1}\left(m_{2}\right) S^{-1}\left(m_{1}\right)\right) h_{2} m_{4} \\
\longrightarrow & 1_{H} \otimes h \otimes m
\end{aligned}
$$

which respects the multiplication with elements of $A$.

We have to show now the exactness of the sequence. For the injectivity of $\widetilde{\rho}_{M}$ : take $m \in M$ such that $m_{0} \otimes \alpha S^{-1}\left(m_{1}\right) m_{2}=0$. Now apply $\rho_{M} \otimes\left[\left(I_{H} \otimes \Delta\right) \Delta\right]$ to get

$$
0=m_{0} \otimes m_{1} \otimes \alpha S^{-1}\left(m_{2}\right) m_{3} \otimes m_{4} \otimes m_{5}
$$

Finally, act on this by $I_{M} \otimes \omega^{321}\left(S^{-1} \otimes I_{H} \otimes \beta S^{-1} \otimes S^{-1}\right)$. By (2.7) it follows $0=m_{0} \varepsilon\left(m_{1}\right)=m$.

Let's check now the exactness in $M \otimes H$. It is straightforward to see that $\left(I_{M} \otimes \widetilde{\Delta}\right) \widetilde{\rho}_{M}=\left(\widetilde{\rho}_{M} \otimes I_{H}\right) \widetilde{\rho}_{M}$. Conversely, let $\sum_{i} m_{i} \otimes h_{i} \in M \otimes H$ such that $\sum_{i} m_{i} \otimes h_{i 1} \otimes \alpha S^{-1}\left(h_{i 2}\right) h_{i 3}=\sum_{i} m_{i 0} \otimes \alpha S^{-1}\left(m_{i 1}\right) m_{i 2} \otimes h_{i}$. 
Apply $\Delta$ and $\left(I_{H} \otimes \Delta\right) \Delta$ on the second, respectively last component, then act by $I_{M} \otimes I_{H} \otimes \omega^{321}\left(S^{-1} \otimes\right.$ $\left.I_{H} \otimes \beta S^{-1} \otimes S^{-1}\right)$. Again using (2.7) we obtain

$$
\begin{aligned}
\sum_{i} m_{i} \otimes h_{i} & =\sum_{i} m_{i 0} \otimes \alpha S^{-1}\left(m_{i 1}\right) m_{i 2} \omega\left(S^{-1}\left(h_{i 3}\right) \beta S^{-1}\left(h_{i 2}\right), h_{i 1}, S^{-1}\left(m_{i 3}\right)\right) \\
& =\widetilde{\rho}_{M}\left(\sum_{i} m_{i 0} \omega\left(S^{-1}\left(h_{i 3}\right) \beta S^{-1}\left(h_{i 2}\right), h_{i 1}, S^{-1}\left(m_{i 1}\right)\right)\right.
\end{aligned}
$$

Therefore the diagram (3.18) is commutative by the naturality of $\varepsilon_{(-)}$. The upper row is exact because $(-)^{c o H}$ is exact and $A$ is a faithfully flat $B$-module. As explained above, $\varepsilon_{M \otimes H}$ and $\varepsilon_{(M \otimes H) \otimes H}$ are bijective, hence $\varepsilon_{M}$ is too, by the Five Lemma.

We move now to the unit $u_{(-)}$of the adjunction. Let $N$ be a right $B$-module. Consider the maps $i_{1}, i_{2}: N \otimes_{B} A \longrightarrow N \otimes_{B} A \otimes_{B} A, i_{1}\left(n \otimes_{B} a\right)=n \otimes_{B} 1_{A} \otimes_{B} a, i_{2}\left(n \otimes_{B} a\right)=n \otimes_{B} a \otimes_{B} 1_{A}$ and the short sequence

$$
0 \longrightarrow N \longrightarrow N \otimes_{B} A \underset{i_{2}}{\stackrel{i_{1}}{\rightrightarrows}} N \otimes_{B} A \otimes_{B} A
$$

where the first morphism is sending $n$ to $n \otimes_{B} 1_{A}$. As $A$ is $B$-flat, this map is injective. Although the associativity of $A$ fails, the faithfully flatness property and the existence of the multiplication and of the unit for $A$ allow us to show, as in the classical case, the exactness of the sequence in the middle term $N \otimes_{B} A$ (we tensor over $B$ one more time with $A$, this is easy to see that is exact, and by faithfully flatness of $A$ we go back to our sequence). Therefore we may consider the diagram with the top row exact

$$
\begin{aligned}
& \begin{array}{ccccc}
N & \longrightarrow & N \otimes_{B} A & \underset{i_{2}}{\stackrel{i_{1}}{\rightrightarrows}} & N \otimes_{B} A \otimes_{B} A \\
& & \| & I_{N} \otimes_{B} \text { can } \downarrow
\end{array} \\
& 0 \longrightarrow\left(N \otimes_{B} A\right)^{c o H} \hookrightarrow N \otimes_{B} A \underset{I_{N} \otimes_{B} I_{A} \otimes u_{H}}{\stackrel{I_{N} \otimes_{B} \bar{\rho}_{A}}{\rightrightarrows}} \quad N \otimes_{B} A \otimes H
\end{aligned}
$$

In the bottom row, the map $\bar{\rho}_{A}$ is given by $\bar{\rho}_{A}(a)=a_{0} \otimes \beta\left(a_{1}\right) a_{2}$. We need to check the exactness of this row. Consider $\sum_{i} n_{i} \otimes_{B} a_{i} \in\left(N \otimes_{B} A\right)^{c o H}$. Then $\sum_{i} n_{i} \otimes_{B} a_{i 0} \otimes a_{i 1}=\sum_{i} n_{i} \otimes_{B} a_{i} \otimes 1_{H}$ implies

$$
\begin{aligned}
\left(I_{N} \otimes_{B} \bar{\rho}_{A}-I_{N} \otimes_{B} I_{A} \otimes u_{H}\right)\left(\sum_{i} n_{i} \otimes_{B} a_{i}\right) & =\sum_{i} n_{i} \otimes_{B} a_{i 0} \otimes \beta\left(a_{i 1}\right) a_{i 2}-\sum_{i} n_{i} \otimes_{B} a_{i} \otimes 1_{H} \\
& =0
\end{aligned}
$$

Conversely, let $\sum_{i} n_{i} \otimes_{B} a_{i} \in N \otimes_{B} A$ which satisfies $\sum_{i} n_{i} \otimes_{B} a_{i 0} \otimes \beta\left(a_{i 1}\right) a_{i 2}=\sum_{i} n_{i} \otimes_{B} a_{i} \otimes 1_{H}$, and apply $\rho_{A}$ and $\left(\Delta \otimes I_{H} \otimes I_{H}\right)\left(\Delta \otimes I_{H}\right) \Delta$ on the second, respectively last component of the tensor product. We obtain

$$
\sum_{i} n_{i} \otimes_{B} a_{i 0} \otimes a_{i 1} \otimes \beta\left(a_{i 2}\right) a_{i 3} \otimes a_{i 4} \otimes a_{i 5} \otimes a_{i 6}=\sum_{i} n_{i} \otimes_{B} a_{i 0} \otimes a_{i 1} \otimes 1_{H} \otimes 1_{H} \otimes 1_{H} \otimes 1_{H}
$$

Now act by $S$ and $\alpha$ on the forth, respectively fifth tensorand and apply $\omega$ on the result. It follows that

$$
\sum_{i} n_{i} \otimes_{B} a_{i 0} \otimes \omega\left(a_{i 1}, \beta\left(a_{i 2}\right) S\left(a_{i 3}\right) \alpha\left(a_{i 4}\right), a_{i 5}\right) a_{i 6}=\sum_{i} n_{i} \otimes_{B} a_{i 0} \otimes \omega\left(a_{i 1}, S\left(1_{H}\right) \alpha\left(1_{H}\right), 1_{H}\right) 1_{H}
$$


meaning

$$
\sum_{i} n_{i} \otimes_{B} a_{i 0} \otimes a_{i 1}=\sum_{i} n_{i} \otimes_{B} a_{i} \otimes 1_{H}
$$

Therefore, the bottom row is exact. The top row is exact by the previous remarks, while the commutativity of the whole diagram can be easily checked. Therefore, $u_{N}$ is bijective by the Five Lemma.

$(2) \Longrightarrow(1)$ Follows as in the Hopf case, using also Corollary (21).

Remark 30. In the proof of the bijectivity of the counit, we have replaced the Galois maps with $\varepsilon_{M} \otimes H$ and $\varepsilon_{(M \otimes H) \otimes H}$. Although in the Hopf algebra case they coincide, in our context the presence of the twist $\widetilde{\mathbf{f}}$ made very difficult to check directly the commutativity of both diagrams. Therefore we have chosen a functorial approach, with appropriately changed morphisms. For the proof of the bijectivity of the second adjunction map $u_{N}$, where $N \in \mathcal{M}_{B}$, a change of morphisms in the horizontal rows was also necessary.

We are going to prove now an affineness condition for coquasi-Hopf algebras. First we need the following

Proposition 31. Let $H$ be a coquasi-Hopf algebra with bijective antipode, $A$ an $H$-comodule algebra and $B=A^{c o H}$. Assume that there exists $\gamma: H \longrightarrow A$ a total integral (i.e. a colinear map satisfying $\left.\gamma\left(1_{H}\right)=1_{A}\right)$. Then $u_{N}: N \longrightarrow\left(N \otimes_{B} A\right)^{c o H}, u_{N}(n)=n \otimes_{B} 1_{A}$, is an isomorphism of right B-modules for all $N \in \mathcal{M}_{B}$.

Proof. We shall define first an analogue of the trace map, namely $t_{A}: A \longrightarrow B, t_{A}(a)=a_{0} \beta\left(a_{1}\right) \gamma S\left(a_{2}\right)$. This is well defined, because

$$
\begin{aligned}
\rho t_{A}(a) & =a_{0_{0}} \beta\left(a_{1}\right) \gamma S\left(a_{2}\right)_{0} \otimes a_{0_{1}} \gamma S\left(a_{2}\right)_{1} \\
(\text { colinearity of } \gamma) & =a_{0} \beta\left(a_{2}\right) \gamma S\left(a_{4}\right) \otimes a_{1} S\left(a_{3}\right) \\
& =a_{0} \beta\left(a_{1}\right) \gamma S\left(a_{2}\right) \otimes 1_{H}
\end{aligned}
$$

where we have used that $S$ is an antimorphism of coalgebras and relation (2.6). Then using again relation (2.6) $)$ one can check that the map $\left(N \otimes_{B} A\right)^{c o H} \longrightarrow N, \sum_{i} n_{i} \otimes_{B} a_{i} \longrightarrow n_{i} t_{A}\left(a_{i}\right)$ is the inverse of $u_{N}$.

We may state now the announced affineness criterion:

Theorem 32. Let $H$ be a coquasi-Hopf algebra with bijective antipode, $A$ an $H$-comodule algebra and $B=$ $A^{\text {coH }}$. Assume that

(1) There exists $\gamma: H \longrightarrow A$ a total integral;

(2) The canonical map can : $A \otimes_{B} A \longrightarrow A \otimes H$ is surjective.

Then the functor of coinvariants and the induction functor form a pair of inverse equivalences between $\mathcal{M}_{A}^{H}$ and $\mathcal{M}_{B}$.

Proof. From the previous Proposition, we know that the unit of the adjunction is bijective. It remains to show that $\varepsilon_{M}: M^{c o H} \otimes_{B} A \longrightarrow M$ is an isomorphism for any Hopf module $M \in \mathcal{M}_{A}^{H}$. We shall follow here the approach from 32 .

Recall that Bulacu and Nauwelaerts ([13]) have proven the equivalence between the existence of a total integral on a comodule algebra $A$ and the injectivity of any Hopf module as a right $H$-comodule. Their result is stated for right Hopf modules, but it holds also for ${ }_{A} \mathcal{M}^{H}$ because the antipode is bijective and $\mathcal{M}_{A}^{H} \simeq{ }_{A_{\mathrm{f}}-1}^{o p} \mathcal{M}^{H^{c o p}}$.

From Remark 24 we know that can is a morphism of left Hopf modules. The composition

$$
\widetilde{\operatorname{can}}: \bullet A^{\bullet} \otimes A \longrightarrow . A^{\bullet} \otimes_{B} A \stackrel{\operatorname{can}}{\longrightarrow} \bullet A^{\bullet} \otimes H^{S, \bullet}
$$


will be a surjective left Hopf module map, therefore it splits as an $H$-comodule map via a colinear morphism $\theta: \bullet A \bullet \otimes H^{S, \bullet} \longrightarrow \bullet A \bullet \otimes$ with $\widetilde{c a n} \theta=I_{A \otimes H}$. Denote $\theta\left(1_{A} \otimes h\right)=\sum_{i} \widetilde{l}_{i}(h) \otimes \widetilde{r}_{i}(h)$ as an extension of the notation from Proposition 23, It follows that analogues of properties (5.1)-(5.4) hold:

$$
\begin{aligned}
\sum_{i} \widetilde{l}_{i}\left(h_{1}\right) \otimes \widetilde{r}_{i}\left(h_{1}\right) \otimes h_{2} & =\sum_{i} \widetilde{l}_{i}(h) \otimes \widetilde{r}_{i}(h)_{0} \otimes \widetilde{r}_{i}(h)_{1} \\
\sum_{i} \widetilde{l}_{i}(h) \widetilde{r}_{i}(h) & =\alpha(h) 1_{A} \\
\sum_{i} \widetilde{l}_{i}(h)_{0} \otimes \widetilde{r}_{i}(h) \otimes \widetilde{l}_{i}(h)_{1} & =\sum_{i} \widetilde{l}_{i}\left(h_{2}\right) \otimes \widetilde{r}_{i}\left(h_{2}\right) \otimes S\left(h_{1}\right) \\
\sum_{i} a_{0} \beta\left(a_{1}\right) \widetilde{l}_{i}\left(a_{2}\right) \otimes \widetilde{r}_{i}\left(a_{2}\right) & =1_{A} \otimes a
\end{aligned}
$$

Relation (3.22) implies $\sum_{i} m_{0} \beta\left(m_{1}\right) \widetilde{l}_{i}\left(m_{2}\right) \otimes \widetilde{r}_{i}\left(m_{2}\right) \in M^{c o H} \otimes A$. Now we can define $\chi_{M}: M \longrightarrow M^{c o H} \otimes_{B} A$, $\chi_{M}(m)=\sum_{i} m_{0} \beta\left(m_{1}\right) \widetilde{l}_{i}\left(m_{2}\right) \otimes_{B} \widetilde{r}_{i}\left(m_{2}\right)$. We claim that this is an inverse for $\varepsilon_{M}$, for any $M \in \mathcal{M}_{A}^{H}$. Indeed

$$
\begin{aligned}
\chi_{M} \varepsilon_{M}\left(m \otimes_{B} a\right) & =\chi_{M}(m a)=\sum_{i} m a_{0} \beta\left(a_{1}\right) \widetilde{l}_{i}\left(a_{2}\right) \otimes_{B} \widetilde{r}_{i}\left(a_{2}\right) \\
& =m \otimes_{B} a
\end{aligned}
$$

for all $m \otimes_{B} a \in M^{c o H} \otimes_{B} A$ and

$$
\begin{aligned}
\varepsilon_{M} \chi_{M}(m) & =\varepsilon_{M}\left(\sum_{i} m_{0} \beta\left(m_{1}\right) \widetilde{l}_{i}\left(m_{2}\right) \otimes_{B} \widetilde{r}_{i}\left(m_{2}\right)\right) \\
& =\sum_{i}\left[m_{0} \beta\left(m_{1}\right) \widetilde{l}_{i}\left(m_{2}\right)\right] \widetilde{r}_{i}\left(m_{2}\right) \\
& =\sum_{i} m_{0}\left[\widetilde{l}_{i}\left(m_{3}\right)_{0} \widetilde{r}_{i}\left(m_{3}\right)_{0}\right] \omega\left(m_{1}, \widetilde{l}_{i}\left(m_{3}\right)_{1}, \widetilde{r}_{i}\left(m_{3}\right)_{1}\right) \beta\left(m_{2}\right) \\
& =\sum_{i} m_{0}\left[\widetilde{l}_{i}\left(m_{4}\right) \widetilde{r}_{i}\left(m_{4}\right)_{0}\right] \omega\left(m_{1}, S\left(m_{3}\right), \widetilde{r}_{i}\left(m_{4}\right)_{1}\right) \beta\left(m_{2}\right) \\
(3.22) & =\sum_{i} m_{0}\left[\widetilde{l}_{i}\left(m_{4}\right) \widetilde{r}_{i}\left(m_{4}\right)\right] \omega\left(m_{1}, S\left(m_{3}\right), m_{5}\right) \beta\left(m_{2}\right) \\
(3.21) & =\sum_{i} m_{0} \omega\left(m_{1}, S\left(m_{3}\right), m_{5}\right) \beta\left(m_{2}\right) \alpha\left(m_{4}\right) \\
& =m
\end{aligned}
$$

for all $m \in M$. It follows that $\varepsilon_{M}$ is bijective.

We can state now all our previous results in the form of the following theorem:

Theorem 33. Let $H$ be a coquasi-Hopf algebra with bijective antipode, $A$ an $H$-comodule algebra and $B=$ $A^{c o H}$. Then the following are equivalent:

(1) There exists a total integral $\gamma: H \longrightarrow A$ and the map can $: A \otimes_{B} A \longrightarrow A \otimes H$ is surjective;

(2) The functor of coinvariants and the induction functor are a pair of inverse equivalences between $\mathcal{M}_{A}^{H}$ and $\mathcal{M}_{B}$; 
(3) The functor of coinvariants and the induction functor are a pair of inverse equivalences between ${ }_{A} \mathcal{M}^{H}$ and ${ }_{B} \mathcal{M}$;

(4) $A$ is faithfully flat as a left $B$-module, and $A$ is a Galois extension of $B$;

(5) $A$ is faithfully flat as a right $B$-module, and $A$ is a Galois extension of $B$.

Proof. (1) $\Longrightarrow$ (2) follows from Theorem 32 (2) $\Longleftrightarrow(4)$ is Theorem 28, (4) $\Longrightarrow$ (1) uses the same argument as in [33, because $c a n^{\prime}$ is also bijective by Lemma14, and is a morphism of left $B$-modules, right $H$-comodules by Proposition 23(6). The sequence of isomorphisms ( $A$ is flat $B$-module)

$$
\left(A^{\bullet} \square_{H} V\right) \otimes_{B} A \simeq\left(A^{\bullet} \otimes_{B} A\right) \square_{H} V \simeq\left(A \otimes H^{\bullet}\right) \square_{H} V \simeq A \otimes V
$$

for each $V \in{ }^{H} \mathcal{M}$, together with the left $B$-faithful flatness of $A$ imply that $A$ is right $H$-coflat, or equivalently, that $A$ is $H$-injective (here $\square_{H}$ is the cotensor product over $H$ ).

$(1) \Longleftrightarrow(3) \Longleftrightarrow(5)$ We simply apply the above to $A^{o p}$ as a right $H^{o p}$-comodule algebra, since the antipode is bijective.

\section{A bialgebroid associated to a faithfully flat Galois extension}

Let $H$ be a coquasi-bialgebra (without any assumption on the antipode) and $A$ a right $H$-comodule algebra. On the tensor product $A \otimes A^{o p}$ we consider the codiagonal right $H$-comodule structure $\rho(a \otimes b)=$ $a_{0} \otimes b_{0} \otimes a_{1} b_{1}$. Denote $L=\left(A \otimes A^{o p}\right)^{c o H}$. Then

Proposition 34. $L$ is an associative $B \otimes B^{o p}$-algebra with unit $1_{A} \otimes 1_{A}$ and multiplication

$$
(a \otimes b)(c \otimes d)=a_{0} c_{0} \otimes d_{0} b_{0} \omega^{-1}\left(a_{1}, c_{1}, d_{1} b_{1}\right) \omega\left(c_{2}, d_{2}, b_{2}\right)
$$

for $a \otimes b, c \otimes d \in L$.

Proof. We shall suppress the $\sum$ symbol when referring to elements of $L$ for simplicity.

After a short calculation, it follows that the multiplication is well-defined, with values in $L$. Moreover, the maps $b \in B \longrightarrow b \otimes 1_{A} \in A \otimes A^{o p}, b \in B^{o p} \longrightarrow 1_{A} \otimes b \in A \otimes A^{o p}$ take values in $L$ and are multiplicative.

It is easy to check that $1_{A} \otimes 1_{A} \in L$ and that it is a unit for the given multiplication. The most difficult part to show is the associativity. Take $a \otimes b, c \otimes d, e \otimes f \in L$ (summation understood). Then we compute

$$
\begin{aligned}
{[(a \otimes b)(c \otimes d)](e \otimes f)=} & \left(a_{0} c_{0} \otimes d_{0} b_{0}\right)(e \otimes f) \omega^{-1}\left(a_{1}, c_{1}, d_{1} b_{1}\right) \omega\left(c_{2}, d_{2}, b_{2}\right) \\
= & \left(a_{0} c_{0}\right) e_{0} \otimes f_{0}\left(d_{0} b_{0}\right) \omega^{-1}\left(a_{1} c_{1}, e_{1}, f_{1}\left(d_{1} b_{1}\right)\right) \omega\left(e_{2}, f_{2}, d_{2} b_{2}\right) \\
& \omega^{-1}\left(a_{2}, c_{2}, d_{3} b_{3}\right) \omega\left(c_{3}, d_{4}, b_{4}\right) \\
= & \left(a_{0} c_{0}\right) e_{0} \otimes\left(f_{0} d_{0}\right) b_{0} \omega^{-1}\left(a_{1} c_{1}, e_{1},\left(f_{1} d_{1}\right) b_{1}\right) \omega^{-1}\left(f_{2}, d_{2}, b_{2}\right) \\
& \omega\left(e_{2}, f_{3}, d_{3} b_{3}\right) \omega^{-1}\left(a_{2}, c_{2}, d_{4} b_{4}\right) \omega\left(c_{3}, d_{5}, b_{5}\right) \\
(2.1) & \left(a_{0} c_{0}\right) e_{0} \otimes\left(f_{0} d_{0}\right) b_{0} \omega^{-1}\left(a_{1} c_{1}, e_{1},\left(f_{1} d_{1}\right) b_{1}\right) \omega\left(e_{2}, f_{2} d_{2}, b_{2}\right) \\
& \omega\left(e_{3}, f_{3}, d_{3}\right) \omega^{-1}\left(e_{4} f_{4}, d_{4}, b_{3}\right) \omega^{-1}\left(a_{2}, c_{2}, d_{5} b_{4}\right) \omega\left(c_{3}, d_{6}, b_{5}\right) \\
(e \otimes f \in L)= & \left(a_{0} c_{0}\right) e_{0} \otimes\left(f_{0} d_{0}\right) b_{0} \omega^{-1}\left(a_{1} c_{1}, e_{1},\left(f_{1} d_{1}\right) b_{1}\right) \omega\left(e_{2}, f_{2} d_{2}, b_{2}\right) \\
& \omega\left(e_{3}, f_{3}, d_{3}\right) \omega^{-1}\left(a_{2}, c_{2}, d_{4} b_{3}\right) \omega\left(c_{3}, d_{5}, b_{4}\right) \\
= & \left(a_{0} c_{0}\right) e_{0} \otimes\left(f_{0} d_{0}\right) b_{0} \omega\left(\left(a_{1} c_{1}\right) e_{1}, f_{1} d_{1}, b_{1}\right) \omega^{-1}\left(a_{2} c_{2}, e_{2}, f_{2} d_{2}\right) \\
& \omega^{-1}\left(a_{3} c_{3}, e_{3}\left(f_{3} d_{3}\right), b_{2}\right) \omega\left(e_{4}, f_{4}, d_{4}\right) \omega^{-1}\left(a_{4}, c_{4}, d_{5} b_{3}\right) \\
& \omega\left(c_{5}, d_{6}, b_{4}\right) \\
= & \left(a_{0} c_{0}\right) e_{0} \otimes\left(f_{0} d_{0}\right) b_{0} \omega\left(\left(a_{1} c_{1}\right) e_{1}, f_{1} d_{1}, b_{1}\right) \omega^{-1}\left(a_{2} c_{2}, e_{2}, f_{2} d_{2}\right) \\
&
\end{aligned}
$$




$$
\begin{aligned}
& \omega\left(e_{3}, f_{3}, d_{3}\right) \omega^{-1}\left(a_{3} c_{3}, d_{4}, b_{2}\right) \omega^{-1}\left(a_{4}, c_{4}, d_{5} b_{3}\right) \omega\left(c_{5}, d_{6}, b_{4}\right) \\
(\underline{2.3}),(c \otimes d \in L)= & \left(a_{0} c_{0}\right) e_{0} \otimes\left(f_{0} d_{0}\right) b_{0} \omega\left(\left(a_{1} c_{1}\right) e_{1}, f_{1} d_{1}, b_{1}\right) \omega^{-1}\left(a_{2} c_{2}, e_{2}, f_{2} d_{2}\right) \\
& \omega\left(e_{3}, f_{3}, d_{3}\right) \omega^{-1}\left(a_{3}, c_{3}, d_{4}\right) \\
(2.1)= & a_{0}\left(c_{0} e_{0}\right) \otimes\left(f_{0} d_{0}\right) b_{0} \omega\left(a_{1}\left(c_{1} e_{1}\right), f_{1} d_{1}, b_{1}\right) \omega\left(a_{2}, c_{2}, e_{2}\right) \\
& \omega^{-1}\left(a_{3} c_{3}, e_{3}, f_{2} d_{2}\right) \omega\left(e_{4}, f_{3}, d_{3}\right) \omega^{-1}\left(a_{4}, c_{4}, d_{4}\right)
\end{aligned}
$$

Apparently this leads nowhere. But let's evaluate also

$$
\begin{aligned}
(a \otimes b)[(c \otimes d)(e \otimes f)]= & (a \otimes b)\left(c_{0} e_{0} \otimes f_{0} d_{0}\right) \omega^{-1}\left(c_{1}, e_{1}, f_{1} d_{1}\right) \omega\left(e_{2}, f_{2}, d_{2}\right) \\
(4.1)= & a_{0}\left(c_{0} e_{0}\right) \otimes\left(f_{0} d_{0}\right) b_{0} \omega^{-1}\left(a_{1}, c_{1} e_{1},\left(f_{1} d_{1}\right) b_{1}\right) \omega\left(c_{2} e_{2}, f_{2} d_{2}, b_{2}\right) \\
& \omega^{-1}\left(c_{3}, e_{3}, f_{3} d_{3}\right) \omega\left(e_{4}, f_{4}, d_{4}\right) \\
= & a_{0}\left(c_{0} e_{0}\right) \otimes\left(f_{0} d_{0}\right) b_{0} \omega\left(a_{1}\left(c_{1} e_{1}\right), f_{1} d_{1}, b_{1}\right) \omega^{-1}\left(a_{2}, c_{2} e_{2}, f_{2} d_{2}\right) \\
& \omega^{-1}\left(a_{3},\left(c_{3} e_{3}\right)\left(f_{3} d_{3}\right), b_{2}\right) \omega^{-1}\left(c_{4}, e_{4}, f_{4} d_{4}\right) \omega\left(e_{5}, f_{5}, d_{5}\right) \\
= & a_{0}\left(c_{0} e_{0}\right) \otimes\left(f_{0} d_{0}\right) b_{0} \omega\left(a_{1}\left(c_{1} e_{1}\right), f_{1} d_{1}, b_{1}\right) \omega^{-1}\left(a_{2}, c_{2} e_{2}, f_{2} d_{2}\right) \\
& \omega^{-1}\left(c_{3}, e_{3}, f_{3} d_{3}\right) \omega\left(e_{4}, f_{4}, d_{4}\right) \omega^{-1}\left(a_{3}, c_{4} d_{5}, b_{2}\right) \\
(c .1), \text { (2.1) } & a_{0}\left(c_{0} e_{0}\right) \otimes\left(f_{0} d_{0}\right) b_{0} \omega\left(a_{1}\left(c_{1} e_{1}\right), f_{1} d_{1}, b_{1}\right) \omega^{-1}\left(a_{2}, c_{2} e_{2}, f_{2} d_{2}\right) \\
& \omega^{-1}\left(c_{3}, e_{3}, f_{3} d_{3}\right) \omega\left(e_{4}, f_{4}, d_{4}\right) \\
= & a_{0}\left(c_{0} e_{0}\right) \otimes\left(f_{0} d_{0}\right) b_{0} \omega\left(a_{1}\left(c_{1} e_{1}\right), f_{1} d_{1}, b_{1}\right) \omega\left(a_{2}, c_{2}, e_{2}\right) \\
& \omega^{-1}\left(a_{3} c_{3}, e_{3}, f_{2} d_{2}\right) \omega^{-1}\left(a_{4}, c_{4}, e_{4}\left(f_{3} d_{3}\right)\right) \omega\left(e_{5}, f_{4}, d_{4}\right) \\
= & a_{0}\left(c_{0} e_{0}\right) \otimes\left(f_{0} d_{0}\right) b_{0} \omega\left(a_{1}\left(c_{1} e_{1}\right), f_{1} d_{1}, b_{1}\right) \omega\left(a_{2}, c_{2}, e_{2}\right) \\
& \omega^{-1}\left(a_{3} c_{3}, e_{3}, f_{2} d_{2}\right) \omega\left(e_{4}, f_{3}, d_{3}\right) \omega^{-1}\left(a_{4}, c_{4}, d_{4}\right) \\
\text { (2.1) }) &
\end{aligned}
$$

hence we have obtained the same as above.

Proposition 35. Let $H$ be a coquasi-Hopf algebra with bijective antipode and $A$ a right $H$-comodule algebra, left faithfully flat and Galois over $B=A^{\mathrm{coH}}$. Then the left L-module category ${ }_{L} \mathcal{M}$ is equivalent to the category of two sided $(H, A)$-Hopf modules ${ }_{A} \mathcal{M}_{A}^{H}$ (i.e. A-bimodules in $\left.\mathcal{M}^{H}\right)$.

Proof. Let $N$ be any left $L$-module. By restriction, $N$ is a left $B^{o p}$-module, that is, a right $B$-module. We can use then the category equivalence $\mathcal{M}_{B} \simeq \mathcal{M}_{A}^{H}$ from Theorem 28, It follows that $N \otimes_{B} A_{:}: \mathcal{M}_{A}^{H}$. But we still need the left $A$-module structure on $N \otimes_{B} A \bullet \bullet$. For this, we shall use the inverse of the Galois map with notations from Proposition 23

$$
a \odot\left(n \otimes_{B} b\right)=\sum_{i}\left[a_{0} \otimes b_{0} \omega\left(a_{1}, b_{1}, \beta\left(a_{2} b_{2}\right) S\left(a_{3} b_{3}\right)\right) l_{i}\left(a_{4} b_{4}\right)\right] n \otimes_{B} r_{i}\left(a_{4} b_{4}\right)
$$

for any $a, b \in A, n \in N$. We should check first if this is well-defined. For this, consider $\left(A^{\bullet} \otimes A^{\bullet}\right) \otimes_{B} A$ as a right $H$-comodule with coaction on the first component. We can compute

$$
\begin{aligned}
\rho\left(\left[a_{0} \otimes b_{0} \omega\left(a_{1}, b_{1}, \beta\left(a_{2} b_{2}\right) S\left(a_{3} b_{3}\right)\right) l_{i}\left(a_{4} b_{4}\right)\right] \otimes_{B} r_{i}\left(a_{4} b_{4}\right)\right)= & \sum_{i}\left[a_{0} \otimes b_{0} \omega\left(a_{2}, b_{2}, \beta\left(a_{3} b_{3}\right) S\left(a_{4} b_{4}\right)\right)\right. \\
& \left.l_{i}\left(a_{5} b_{5}\right)_{0}\right] \otimes_{B} r_{i}\left(a_{6} b_{6}\right) \otimes a_{1}\left[b_{1} l_{i}\left(a_{5} b_{5}\right)_{1}\right] \\
(\text { Proposition 23(5.3)), (2.1), (2.6) }= & \sum_{i}\left[a_{0} \otimes b_{0} \omega\left(a_{1}, b_{1}, \beta\left(a_{2} b_{2}\right) S\left(a_{3} b_{3}\right)\right)\right. \\
& \left.l_{i}\left(a_{4} b_{4}\right)\right] \otimes_{B} r_{i}\left(a_{4} b_{4}\right) \otimes 1_{H}
\end{aligned}
$$


By the left $B$-flatness of $A$, it follows that

$$
\sum_{i}\left[a_{0} \otimes b_{0} \omega\left(a_{1}, b_{1}, \beta\left(a_{2} b_{2}\right) S\left(a_{3} b_{3}\right)\right) l_{i}\left(a_{4} b_{4}\right)\right] \otimes_{B} r_{i}\left(a_{4} b_{4}\right) \in\left(A^{\bullet} \otimes A^{\bullet}\right)^{c o H} \otimes_{B} A
$$

Therefore, relation (4.2) is correct. We still need to check that the formula defines on $N \otimes_{B} A$ : an $A$-bimodule structure in $\mathcal{M}^{H}$. The left $A$-multiplication is $H$-colinear and

$$
\begin{aligned}
1_{A} \odot\left(n \otimes_{B} b\right) & =\sum_{i}\left[1_{A} \otimes b_{0} \beta\left(b_{1}\right) l_{i}\left(b_{2}\right)\right] n \otimes_{B} r_{i}\left(b_{2}\right) \\
(\text { Proposition 23 }(5.4)) & =\left(1_{A} \otimes 1_{A}\right) n \otimes_{B} b \\
& =n \otimes_{B} b
\end{aligned}
$$

Now we compute

$$
\begin{aligned}
& a_{0} \odot\left(b_{0} \odot\left(n \otimes_{B} c_{0}\right)\right) \omega\left(a_{1}, b_{1}, c_{1}\right)=\sum_{i} a_{0} \odot\left[\left[b_{0} \otimes c_{0} \omega\left(b_{1}, c_{1}, \beta\left(b_{2} c_{2}\right) S\left(b_{3} c_{3}\right)\right)\right.\right. \\
& \left.\left.l_{i}\left(b_{4} c_{4}\right)\right] n \otimes_{B} r_{i}\left(b_{4} c_{4}\right)\right] \omega\left(a_{1}, b_{5}, c_{5}\right) \\
& (\text { Proposition 23 }(5.1))=\sum_{i, j}\left(a_{0} \otimes r_{i}\left(b_{4} c_{4}\right) l_{j}\left(a_{4}\left(b_{8} c_{8}\right)\right)\right)\left(b_{0} \otimes c_{0} l_{i}\left(b_{4} c_{4}\right)\right) n \\
& \otimes_{B} r_{j}\left(a_{4}\left(b_{8} c_{8}\right)\right) \omega\left(a_{1}, b_{5} c_{5}, S\left(a_{3}\left(b_{7} c_{7}\right)\right)\right. \\
& \omega\left(b_{1}, c_{1}, S\left(b_{3} c_{3}\right)\right) \beta\left(b_{2} c_{2}\right) \beta\left(a_{2}\left(b_{6} c_{6}\right)\right) \\
& \text { (4.1), (Proposition 23)(5.1),(5.3)) }=\sum_{i, j}\left\{a_{0} b_{0} \otimes\left[c_{0} l_{i}\left(b_{8} c_{8}\right)\right]\left[r_{i}\left(b_{8} c_{8}\right) l_{j}\left(a_{7}\left(b_{16} c_{16}\right)\right)\right]\right\} n \\
& \otimes_{B} r_{j}\left(a_{7}\left(b_{16} c_{16}\right)\right) \omega^{-1}\left(a_{1}, b_{1},\left[c_{1} S\left(b_{7} c_{7}\right)\right]\left[\left(b_{9} c_{9}\right)\right.\right. \\
& \left.\left.S\left(a_{6}\left(b_{15} c_{15}\right)\right)\right]\right) \omega\left(b_{2}, c_{2} S\left(b_{6} c_{6}\right),\left(b_{10} c_{10}\right)\right. \\
& \left.S\left(a_{5}\left(b_{14} c_{14}\right)\right)\right) \omega\left(a_{2}, b_{11} c_{11}, S\left(a_{4}\left(b_{13} c_{13}\right)\right)\right) \\
& \omega\left(b_{3}, c_{3}, S\left(b_{5} c_{5}\right)\right) \beta\left(b_{4} c_{4}\right) \beta\left(a_{3}\left(b_{12} c_{12}\right)\right) \\
& \text { (2.3), (2.1), (2.25) }=\sum_{i, j}\left\{a_{0} b_{0} \otimes c_{0}\left[l_{i}\left(b_{8} c_{8}\right)\left[r_{i}\left(b_{8} c_{8}\right) l_{j}\left(a_{8}\left(b_{18} c_{18}\right)\right)\right]\right]\right\} n \\
& \otimes_{B} r_{j}\left(a_{8}\left(b_{18} c_{18}\right)\right) \omega^{-1}\left(a_{1}, b_{1}, c_{1}\left[S ( b _ { 7 } c _ { 7 } ) \left[\left(b_{9} c_{9}\right)\right.\right.\right. \\
& \left.\left.\left.S\left(a_{7}\left(b_{17} c_{17}\right)\right)\right]\right]\right) \omega\left(b_{2}, c_{2}, S\left(b_{6} c_{6}\right)\left[\left(b_{10} c_{10}\right)\right.\right. \\
& \left.\left.S\left(a_{6}\left(b_{16} c_{16}\right)\right)\right]\right) \omega\left(b_{3} c_{3}, S\left(b_{5} c_{5}\right),\left(b_{11} c_{11}\right)\right. \\
& \left.S\left(a_{5}\left(b_{15} c_{15}\right)\right)\right) \omega\left(a_{2}, b_{12} c_{12}, S\left(a_{4}\left(b_{14} c_{14}\right)\right)\right) \\
& \beta\left(b_{4} c_{4}\right) \beta\left(a_{3}\left(b_{13} c_{13}\right)\right) \\
& \text { (2.1), (2.25), (Proposition 23)(5.2)), (2.5) }=\sum_{j}\left\{a_{0} b_{0} \otimes c_{0} l_{j}\left(a_{9}\left(b_{17} c_{17}\right)\right)\right\} n \otimes_{B} r_{j}\left(a_{9}\left(b_{17} c_{17}\right)\right) \\
& \omega^{-1}\left(a_{1}, b_{1}, c_{1} S\left(a_{8}\left(b_{16} c_{16}\right)\right)\right) \\
& \omega\left(b_{2}, c_{2}, S\left(a_{7}\left(b_{15} c_{15}\right)\right)\right) \\
& \omega^{-1}\left(S\left(b_{6} c_{6}\right), b_{8} c_{8}, S\left(a_{6}\left(b_{14} c_{14}\right)\right)\right) \omega\left(b_{3} c_{3}, S\left(b_{5} c_{5}\right),\right. \\
& \left.\left(b_{9} c_{9}\right) S\left(a_{5}\left(b_{13} c_{13}\right)\right)\right) \omega\left(a_{2}, b_{10} c_{10}, S\left(a_{4}\left(b_{12} c_{12}\right)\right)\right) \\
& \beta\left(b_{4} c_{4}\right) \beta\left(a_{3}\left(b_{11} c_{11}\right)\right) \\
& \text { (2.3), (2.5), (2.6), (2.7) }=\sum_{j}\left\{a_{0} b_{0} \otimes c_{0} l_{j}\left(a_{7}\left(b_{8} c_{8}\right)\right)\right\} n \otimes_{B} r_{j}\left(a_{7}\left(b_{8} c_{8}\right)\right)
\end{aligned}
$$




$$
\begin{aligned}
& \omega^{-1}\left(a_{1}, b_{1}, c_{1} S\left(a_{6}\left(b_{7} c_{7}\right)\right)\right) \omega\left(b_{2}, c_{2}, S\left(a_{5}\left(b_{6} c_{6}\right)\right)\right) \\
& \omega\left(a_{2}, b_{3} c_{3}, S\left(a_{4}\left(b_{5} c_{5}\right)\right)\right) \beta\left(a_{3}\left(b_{4} c_{4}\right)\right) \\
(\underline{2.3}),(2.1)= & \sum_{j}\left\{a_{0} b_{0} \otimes c_{0} l_{j}\left(\left(a_{4} b_{4}\right) c_{4}\right)\right\} n \otimes_{B} r_{j}\left(\left(a_{4} b_{4}\right) c_{8}\right) \\
& \omega\left(a_{1} b_{1}, c_{1} S\left(\left(a_{3} b_{3}\right) c_{3}\right) \beta\left(\left(a_{2} b_{2}\right) c_{2}\right)\right. \\
= & (a b) \odot\left(n \otimes_{B} c\right)
\end{aligned}
$$

hence $N \otimes_{B} A$ is a left $A$-module in $\mathcal{M}^{H}$. We only have to show the compatibility between the two $A$-module structures:

$$
\begin{aligned}
& a_{0} \odot\left[\left(n \otimes_{B} b_{0}\right) c_{0}\right] \omega\left(a_{1}, b_{1}, c_{1}\right)=\sum_{i}\left[a_{0} \otimes\left(b_{0} c_{0}\right) l_{i}\left(a_{4}\left(b_{4} c_{4}\right)\right)\right] n \otimes_{B} r_{i}\left(a_{4}\right. \\
& \left.\left(b_{4} c_{4}\right)\right) \omega\left(a_{1}, b_{1} c_{1}, S\left(a_{3}\left(b_{3} c_{3}\right)\right) \omega\left(a_{5}, b_{5}, c_{5}\right)\right. \\
& \beta\left(a_{2}\left(b_{2} c_{2}\right)\right) \\
& \text { (2.1), (Proposition 23) (5.3), (5.5)), (2.15), (2.19) }=\sum_{i, j}\left[a_{0} \otimes\left(b_{0} c_{0}\right) l_{i}\left(c_{5}\right) l_{j}\left(a_{5} b_{5}\right)\right] n \otimes_{B} r_{j}\left(a_{5} b_{5}\right) \\
& r_{i}\left(c_{5}\right) \omega\left(a_{1}, b_{1} c_{1}, S\left(c_{4}\right) S\left(a_{4} b_{4}\right)\right) \omega\left(a_{2}, b_{2}, c_{2}\right) \\
& q\left(a_{3} b_{3}, c_{3}\right) \\
& \text { (2.17), (2.25), (2.25), (Proposition 23) (5.3)) }=\sum_{i, j}\left[a_{0} \otimes b_{0}\left\{\left[c_{0} l_{i}\left(c_{13}\right)\right] l_{j}\left(a_{10} b_{11}\right)\right\}\right] n \otimes_{B} \\
& r_{j}\left(a_{10} b_{11}\right) r_{i}\left(c_{13}\right) \omega^{-1}\left(c_{1}, S\left(c_{12}\right), S\left(a_{9} b_{10}\right)\right) \\
& \omega\left(b_{1}, c_{2}, S\left(c_{11}\right) S\left(a_{8} b_{9}\right)\right) \\
& \omega\left(a_{1}, b_{2} c_{3}, S\left(c_{10}\right) S\left(a_{7} b_{8}\right)\right) \\
& \omega\left(a_{2}, b_{3}, c_{4}\right) \omega\left(\left(a_{3} b_{4}\right) c_{5}, S\left(c_{9}\right), S\left(a_{6} b_{7}\right)\right) \\
& \omega^{-1}\left(a_{4} b_{5}, c_{6}, S\left(c_{8}\right)\right) \beta\left(a_{5} b_{6}\right) \beta\left(c_{7}\right) \\
& \text { (2.3), (2.3), (2.1), (2.6) }=\sum_{i, j}\left[a_{0} \otimes b_{0}\left\{\left[c_{0} l_{i}\left(c_{2}\right)\right] l_{j}\left(a_{4} b_{4}\right)\right\}\right] n \otimes_{B} \\
& r_{j}\left(a_{4} b_{4}\right) r_{i}\left(c_{2}\right) \omega\left(a_{1}, b_{1}, S\left(a_{3} b_{3}\right)\right) \\
& \beta\left(a_{2} b_{2}\right) \beta\left(c_{1}\right) \\
& (\text { Proposition 23 }(5.4))=\left(a \odot\left(n \otimes_{B} b\right)\right) c
\end{aligned}
$$

Hence $N \otimes_{B} A \in{ }_{A} \mathcal{M}_{A}^{H}$. It is easy to see that a map $L$-linear $\eta: N_{1} \longrightarrow N_{2}$ induces a morphism $\eta \otimes_{B} I_{A}$ in ${ }_{A} \mathcal{M}_{A}^{H}$. We get then a functor $\mathcal{F}:{ }_{L} \mathcal{M} \longrightarrow{ }_{A} \mathcal{M}_{A}^{H}$. For the inverse construction, let $M \in{ }_{A} \mathcal{M}_{A}^{H}$. Then $M^{c o H} \in \mathcal{M}_{B}={ }_{B^{o p}} \mathcal{M}$. For any $m \in M^{c o H}$ and $a \otimes b \in L$, we may define

$$
(a \otimes b) m=a(m b) \stackrel{m \in \underline{M}^{c o H}}{=}(a m) b
$$

Using this multiplication, $M^{c o H} \in{ }_{L} \mathcal{M}$ and we have a functor $\mathcal{G}:{ }_{A} \mathcal{M}_{A}^{H} \longrightarrow{ }_{L} \mathcal{M}$.

Notice that these two functors are obtained simply restricting the ones in Theorem 28 . The unit and the counit are easily checked to be morphisms in the restricted categories. Therefore we get the category equivalence ${ }_{L} \mathcal{M} \simeq{ }_{A} \mathcal{M}_{A}^{H}$.

Corollary 36. The category ${ }_{L} \mathcal{M}$ is monoidal.

Proof. As ${ }_{A} \mathcal{M}_{A}^{H}$ is monoidal with $\bigcirc_{A}$ the tensor product over $A$ in the comodule category, it remains only to transport the monoidal structure. 
Remark 37. The previous theorem generalizes Schauenburg's result in the Hopf algebra case ([30]). In [31, he gave a categorical proof, using actions of monoidal categories. All his arguments were purely categorical, explaining why Schauenburg's construction can also be performed for coquasi-Hopf algebras. But in order to avoid long and tedious computations, we preferred the direct approach.

In [30 it was also shown that there is a $\times_{B}$-bialgebra (in the sense of Takeuchi) structure on $L$, using precisely the monoidal structure given by the above corollary. Let see now that a similar result holds also in the coquasi case. But first we have an inconvenient: we cannot tensor over $A$, as this is not an associative algebra. This can be avoided by considering suitable tensor product, namely in the monoidal category of comodules.

Lemma 38. Let $H$ be a coquasi-bialgebra, $A$ a right $H$-comodule algebra and $B=A^{c o H}$. For any right $B$-module $N$ and any left Hopf module $M \in{ }_{A} \mathcal{M}^{H}$, we have $\left(N \otimes_{B} A_{\bullet}\right) \bigcirc_{A} M^{\bullet} \simeq N \otimes_{B} M^{\bullet}$ as comodules, where $\bigcirc_{A}$ denotes the tensor product over $A$ in the monoidal category $\mathcal{M}^{H}, N \otimes_{B} A:$ is the induced right Hopf module and $N \otimes_{B} M^{\bullet}$ carries the comodule structure given by that of $M$.

Proof. Recall that the tensor product over $A$ is the equalizer (in the category of right comodules) of the following morphisms $j_{1}, j_{2}:\left[\left(N \otimes_{B} A\right) \otimes A\right] \otimes M \longrightarrow\left(N \otimes_{B} A\right) \otimes M$, where

$$
\begin{aligned}
& j_{1}\left(\left[\left(n \otimes_{B} a\right) \otimes b\right] \otimes m=n \otimes_{B} a b \otimes m\right. \\
& j_{1}\left(\left[\left(n \otimes_{B} a\right) \otimes b\right] \otimes m=n \otimes_{B} a_{0} \otimes b_{0} m_{0} \omega\left(a_{1}, b_{1}, m_{1}\right)\right.
\end{aligned}
$$

Now define $\varphi:\left(N \otimes_{B} A\right) \otimes M \longrightarrow N \otimes_{B} M, \varphi\left(\left(n \otimes_{B} a\right) \otimes m\right)=n \otimes_{B} a m$. Then $\varphi$ is colinear and $\varphi j_{1}=\varphi j_{2}$. Hence it induces the desired isomorphism.

Corollary 39. Let $H$ be a coquasi-Hopf algebra with bijective antipode and $A$ a right $H$-comodule algebra, left faithfully flat and Galois over $B=A^{c o H}$. Then the equivalence $\mathcal{M}_{A}^{H} \simeq \mathcal{M}_{B}$ induces a monoidal functor $(-)^{c o H}:{ }_{A} \mathcal{M}_{A}^{H} \simeq{ }_{B} \mathcal{M}_{B}$.

Proof. It follows by the previous Lemma and from [30, Lemma 6.1.

As the monoidal structure of ${ }_{L} \mathcal{M}$ comes from the one of ${ }_{A} \mathcal{M}_{A}^{H}$ and the functorial diagram

$$
\begin{array}{ccc}
{ }_{A} \mathcal{M}_{A}^{H} \\
(-)^{c o H} \searrow & \rightleftarrows & { }_{B^{\circ p} \mathcal{M}} \mathcal{M} \\
& \swarrow \mathcal{U}
\end{array}
$$

commutes, where $\mathcal{U}$ is the forgetful functor, from Corollary 36 it follows that there $\mathcal{U}$ is also monoidal. But

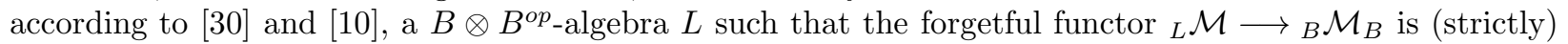
monoidal is precisely a $\times_{B}$-bialgebra (in the sense of Takeuchi) or equivalently, a bialgebroid. Therefore we have obtained a new structure object $L$, whose properties (mainly for the case $B=\mathbb{k}$ ) will make the purpose of an author's forthcoming paper. Having in mind the Hopf algebra case, where the biGalois extensions and torsors are involved, it is expected that this will clarify more about the connections between various generalizations of Hopf algebras.

Acknowledgement. The author would like to thank Prof. C. Năstăsescu and F. Panaite for their useful comments which improved this paper. 


\section{References}

[1] H. Albuquerque and S. Majid. Quasialgebra Structure of the Octonions. J. Algebra, 220(1):188-224, 1999.

[2] H. Albuquerque and S. Majid. $\mathbb{Z}_{n}$-Quasialgebras. In Matrices and Group Representations. Proceedings of a Workshop Dedicated to Professor Graciano N. De Oliveira on the Occasion of his 60th Birthday, Coimbra, Portugal, May 6-8, 1998, volume 19 of Textos Math., Ser., pages 57-64. Coimbra: Univ. de Coimbra, Departamento de Matemática, 1999.

[3] D. Altschuler and A. Coste. Quasi-Quantum groups, Knots, Three Manifolds and Topological Field Theory. Comm. Math. Phys., 150:83-107, 1992.

[4] A. Ardizzoni, C. Menini, and D. Stefan. Hochschild Cohomology and Smoothness in Monoidal Categories. J. Pure Appl. Algebra, 208:297-330, 2007.

[5] A. Balan. Crossed Products for Coquasi-Hopf Algebras. preprint 2007.

[6] A. Balan. A Morita Context and Galois Extensions for Quasi-Hopf Algebras. to appear in Comm. Alg.; available on arXiv:math.QA/0705.3515, 2007.

[7] R. J. Blattner and S. Montgomery. Crossed Products and Galois Extensions of Hopf Algebras. Pacific J. Math., 137:37-54, 1989.

[8] G. Böhm. Galois Theory for Hopf Algebroids. Ann. Univ. Ferrara, Nuova Ser., Sez. VII, 51:233-262, 2005.

[9] T. Brzèzinski and P. M. Hajac. Coalgebra Extensions and Algebra Coextensions of Galois Type. Comm. Algebra, 27:1347-1367, 1999.

[10] T. Brzezinski and G. Militaru. Bialgebroids, $\times_{A}$-Bialgebras and Duality. J. Algebra, 251(1):279-294, 2002.

[11] D. Bulacu. On the Antipode of Semi-Hopf Algebras and Braided Semi-Hopf Algebras. Rev. Roum. Math. Pures Appl., 44(3):329-340, 1999.

[12] D. Bulacu and B. Chirita. Dual Drinfeld Double by Diagonal Crossed Product. Rev. Roum. Math. Pures Appl., 47(3):271-294, 2002.

[13] D. Bulacu and E. Nauwelaerts. Relative Hopf Modules for (Dual) Quasi-Hopf Algebras. J. Algebra, $229(2): 632-659,2000$.

[14] D. Bulacu and E. Nauwelaerts. Dual Quasi-Hopf Algebra Coactions, Smash Coproducts and Relative Hopf Modules. Rev. Roum. Math. Pures Appl., 47(4):415-443, 2002.

[15] S. U. Chase, D. K. Harrison, and A. Rosenberg. Galois Theory and Cohomology of Commutative Rings. Number 52 in AMS Memoirs. AMS, Providence, R. I., 1965.

[16] S. U. Chase and M. E. Sweedler. Hopf Algebras and Galois Theory. Number 97 in Lect. Notes in Math. Springer Verlag, Berlin, 1969.

[17] Y. Doi and M. Takeuchi. Cleft Comodule Algebras for a Bialgebra. Comm. Alg., 14:801-818, 1986.

[18] Y. Doi and M. Takeuchi. Hopf-Galois Extensions of Algebras, the Miyashita-Ulbrich Action, and Azumaya Algebras. J. Algebra, 121(2):488-516, 1989. 
[19] V. G. Drinfeld. Quasi-Hopf Algebras. Leningrad Math. J., 1:1419-1457, 1990.

[20] S. Dăscălescu, C. Năstăsescu, and Ş. Raianu. Hopf Algebras: An Introduction. Number 235 in Pure and Applied Math. Marcel Dekker, New York, 2001.

[21] L. Kadison and K. Szlachanyi. Bialgebroid Actions on Depth Two Extensions and Duality. Adv. Math., 179:75-121, 2003.

[22] C. Kassel. Quantum Groups. Number 155 in Graduate Texts in Math. Springer Verlag, 1995.

[23] H. F. Kreimer and M. Takeuchi. Hopf Algebras and Galois Extensions of an Algebra. Indiana Math. J., 30:675-692, 1981.

[24] S. Majid. Tannaka-Krein Theorem for Quasi-Hopf Algebras. In M. Gestenhaber and J. Stasheff, editors, Deformation Theory and Quantum Groups with Applications to Mathematical Physics, Amherst, MA, 1990, volume 134 of Contemp. Math., pages 219-232. Amer. Math. Soc., Providence, RI, 1992.

[25] S. Majid. Foundations of Quantum Group Theory. Cambridge University Presss, Cambridge, 1995.

[26] A. Masuoka. More Homological Approach to Composition of Subfactors. J. Math. Sci., Tokyo, 10(4):599-630, 2003.

[27] F. Panaite and D. Ştefan. When is the Category of Comodules a Braided Tensor Category? Rev. Roum. Math. Pures Appl., 42(1-2):107-119, 1997.

[28] B. Pareigis. Non-Additive Ring and Module Theory I. General Theory of Monoids. Publ. Math. Debrecen, 24:189-204, 1977.

[29] B. Pareigis. Non-Additive Ring and Module Theory II. C-Categories, C-Functors and C-Morphisms. Publ. Math. Debrecen, 24:351-361, 1977.

[30] P. Schauenburg. Bialgebras over Noncommutative Rings and a Structure Theorem for Hopf Bimodules. Appl. Cat. Str., 6:193-222, 1998.

[31] P. Schauenburg. Actions of Monoidal Categories, and Generalized Hopf Smash Products. J. Alg., 270:521-563, 2003.

[32] P. Schauenburg. Hopf-Galois and Bi-Galois Extensions. In G. Janelidze, B. Pareigis, and W. Tholen, editors, Galois Theory, Hopf Algebras, and Semiabelian Categories, volume 43 of Fields Inst. Commun. AMS, 2004.

[33] H.-J. Schneider. Principal Homogeneous Spaces for Arbitrary Hopf Algebras. Israel J. Math., 72(12):167-195, 1990.

[34] H.-J. Schneider. Representation Theory of Hopf Galois Extensions. Israel J. Math., 72(1-2):196-231, 1990.

[35] K. H. Ulbrich. Vollgraduierte Algebren. Abh. Math. Sem. Univ. Hamburg, 51:136-148, 1981. 